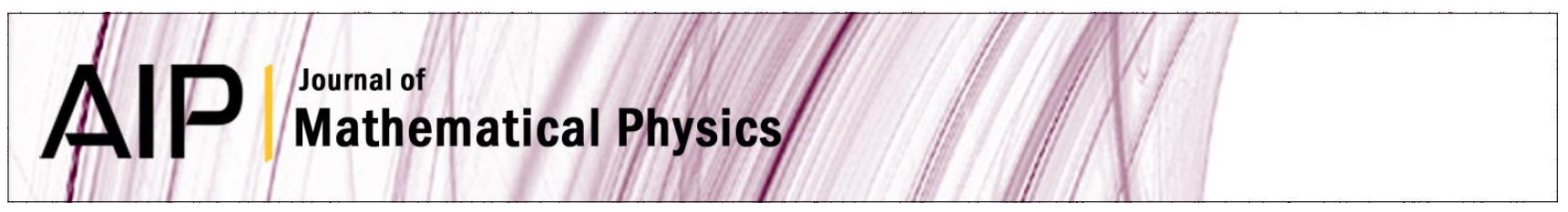

\title{
On form factors of the conjugated field in the nonlinear Schrödinger model
} K. K. Kozlowski

Citation: J. Math. Phys. 52, 083302 (2011); doi: 10.1063/1.3625628

View online: http://dx.doi.org/10.1063/1.3625628

View Table of Contents: http://jmp.aip.org/resource/1/JMAPAQ/v52/i8

Published by the American Institute of Physics.

\section{Related Articles}

Group-theoretical derivation of Aharonov-Bohm phase shifts J. Math. Phys. 54, 021703 (2013)

The equivalence of the Chern-Simons-Schrödinger equations and its self-dual system J. Math. Phys. 54, 021502 (2013)

Symmetry classification of variable coefficient cubic-quintic nonlinear Schrödinger equations J. Math. Phys. 54, 023502 (2013)

The Schrödinger equation with friction from the quantum trajectory perspective J. Chem. Phys. 138, 054107 (2013)

The existence of steady solutions for a class of Schrödinger equations in nonlinear optical lattices J. Math. Phys. 54, 011505 (2013)

\section{Additional information on J. Math. Phys.}

Journal Homepage: http://jmp.aip.org/

Journal Information: http://jmp.aip.org/about/about_the_journal

Top downloads: http://jmp.aip.org/features/most_downloaded

Information for Authors: http://jmp.aip.org/authors

\section{ADVERTISEMENT}

The most comprehensive support for physics in any mathematical software package World-leading tools for performing calculations in theoretical physics

www.maplesoft.com/physics
Your work in Maple matches how you would write the problems and solutions by hand

- State-of-the-art environment for algebraic computations in physics

The only system with the ability to handle a wide range of physics computations as well as pencil-and-paper style input and textbook-quality display of results

- Access to Maple's full mathematical power, programming language, visualization routines, and document creation tools 


\title{
On form factors of the conjugated field in the nonlinear Schrödinger model
}

\author{
K. K. Kozlowskia) \\ DESY, Notkestrasse 85, Hamburg 22607, Germany
}

(Received 5 May 2011; accepted 24 July 2011; published online 16 August 2011)

\begin{abstract}
Izergin-Korepin's lattice discretization of the nonlinear Schrödinger model along with Oota's inverse problem provides one with determinant representations for the form factors of the lattice discretized conjugated field operator. We prove that these form factors converge, in the zero lattice spacing limit, to those of the conjugated field operator in the continuous model. We also compute the large-volume asymptotic behavior of such form factors in the continuous model. These are, in particular, characterized by Fredholm determinants of operators acting on closed contours. We provide a way of defining these Fredholm determinants in the case of generic parameters. @ 2011 American Institute of Physics. [doi:10.1063/1.3625628]
\end{abstract}

\section{INTRODUCTION}

Finite volume lattice discretizations provide a natural way of circumventing problems related with the ultraviolet and infrared divergencies of quantum field theories in infinite volume. As such, they offer a possibility of a rigorous analysis of the spectrum and correlation functions, the strategy being first to obtain expressions for the lattice discretized finite-volume model and then take appropriate limits so as to reach the results relative to the continuous models of quantum field theory in infinite volume. Clearly, in general, carrying out such a program is hopeless in as much as finite-volume lattice discretizations introduce tremendous complication of the model. Yet, in the case of integrable quantum field theories in $(1+1)$ dimensions it has been shown that, for a wide variety of models, there do indeed exist finite volume lattice discretizations preserving the integrable structure of the model. ${ }^{1,6,18}$ The latter can be solved either by means of the algebraic Bethe Ansatz ${ }^{3,6}$ or through the quantum separation of variables. ${ }^{1,3,16}$ Such methods lead eventually to the characterization of the spectrum by means of nonlinear integral equations. ${ }^{4,23}$ It is then possible to take the continuous (infinite number of sites) limit on the level of such nonlinear integral equations. This gives access to the spectrum of the associated quantum field theory infinite volume. In such a way, it was shown for several models ${ }^{4,15}$ that the infinite volume limit of such a description reproduces the predictions ${ }^{24}$ for the $S$ matrix and the spectrum that were building on the factorizable scattering theory in infinite volume.

The purpose of this paper is to push the study of continuous limits of integrable lattice regularizations of quantum field theories a step further, this time in respect to the correlation functions. We will focus on the simplest possible example, the nonlinear Schrödinger model (NLSM). Starting from its lattice discretization introduced by Izering and Korepin, ${ }^{6}$ we recall the inverse problem of Oota ${ }^{17}$ and Slavnov's scalar product formula ${ }^{21}$ so as to provide determinant representations for the lattice approximation of the conjugated field operator. By generalizing and simplifying the approach of Ref. 5, we show that these form factors, along with the generic scalar products and norms, converge, when the lattice spacing goes to zero, to the associated quantities arising in the continuous model in finite volume $L$. This constitutes the main result of the paper. Our approach can be applied to many other correlators in this model. In particular, it provides the missing steps in the derivation of the previously obtained determinant representations for the field, conjugate field,

a)Electronic mail: karol.kajetan.kozlowski@desy.de. 
and current operators ${ }^{12,17}$ in the continuous model. Finally, building on the techniques introduced in Ref. 22 and further developed in Refs. 7 and 9, we compute the large volume $L$ behavior of the properly normalized determinant representation for the conjugated field's form factors. These results are alternative to those obtained in Ref. 2 in as much as we start from different determinant representations. The large volume asymptotics, we obtain are used in Ref. 13 to derive the long-time and large-distance asymptotic behavior of the so-called one particle reduced density matrix in the NLSM. We also would like to recall that such large $L$ asymptotics of properly normalized form factors involve Fredholm determinants of integral operators acting on a closed contour. These determinants may fail to be well defined in the case of arbitrary excited state. In the core of this paper we provide a way to circumvent such difficulties.

This paper is organized as follows. In Sec. I, we introduce the lattice discretization of the NLSM and recall several known facts about the model. In Sec. II, we present the main result of the paper: the convergence (in the zero lattice spacing limit) of the form factors for the lattice discretization of the model to those of the continuous model. We also provide determinant representations for these form factors in the continuum. In Sec. III, we provide formulas for the large volume limit for these form factors in the so-called $n$-particle/hole sector. In addition, we prove a theorem providing some clarification in respect to the definition of the Fredholm determinants occurring in these expressions. The proof of Theorem 2.2, which is slightly technical, is gathered in Appendix.

\section{THE LATTICE DISCRETIZATION OF THE MODEL}

\section{A. The Lax matrix}

The Lax matrix proposed by Izergin and Korepin ${ }^{6}$ for the lattice nonlinear Schrödinger model reads

$L_{0 n}(\lambda)=\left(\begin{array}{cc}-i \frac{\lambda}{2} \Delta+Z_{n}+c \chi_{n}^{*} \chi_{n} / 2 & -i \sqrt{c} \chi_{n}^{*} \rho_{Z_{n}} \\ i \sqrt{c} \rho_{Z_{n}} \chi_{n} & i \frac{\lambda}{2} \Delta+Z_{n}+c \chi_{n}^{*} \chi_{n} / 2\end{array}\right)$, where $\quad Z_{n}=1+(-1)^{n} c \Delta / 4$.

It is represented as a $2 \times 2$ matrix on the auxiliary space $V_{0} \simeq \mathbb{C}^{2}$ whose entries are operators acting on some dense subspace of $\mathcal{H}_{n} \simeq L^{2}(\mathbb{R})$. The operators $\chi_{n}, \chi_{m}^{*}$ are canonical Bose fields with commutation relations $\left[\chi_{n}, \chi_{m}^{*}\right]=\Delta \delta_{n, m}$. In particular, $\chi_{n}^{*}$ is the adjoint of $\chi_{n}$ and $\rho_{Z_{n}}$ $=\sqrt{Z_{n}+c \chi_{n}^{*} \chi_{n} / 4}$. The parameter $\Delta$ plays the role of the lattice spacing.

The index $n$ labels the copy of the quantum space $\mathcal{H}_{n}$, where the canonical fields $\chi_{n}, \chi_{n}^{*}$ act non-trivially. It is readily checked that the various fields entering in the definition of the Lax matrix satisfy to the additional relations

$$
\chi_{n} \rho_{Z_{n}-\frac{\Delta c}{4}}=\rho_{Z_{n}} \chi_{n} \quad \text { and } \quad \rho_{Z_{n}-\frac{\Delta c}{4}} \chi_{n}^{*}=\chi_{n}^{*} \rho_{Z_{n}} .
$$

The Lax matrix (2.1) satisfies the Yang-Baxter equation

$$
R_{00^{\prime}}(\lambda-\mu) L_{0 n}(\lambda) L_{0^{\prime} n}(\mu)=L_{0^{\prime} n}(\mu) L_{0 n}(\lambda) R_{00^{\prime}}(\lambda-\mu),
$$

driven by the rational R-matrix $R_{00^{\prime}}(\lambda)=\lambda-i c \mathcal{P}_{00^{\prime}}$, with $\mathcal{P}_{00^{\prime}}$ being the permutation operator in $V_{0} \otimes V_{0^{\prime}}$. The matrix $R(\lambda)$ becomes a one-dimensional projector at $\lambda=i c$. As a consequence, the Lax matrix $L_{0 n}(\lambda)$ satisfies the quantum determinant relation

$$
\begin{aligned}
L_{0 n}(\lambda) \sigma_{0}^{y} L_{0 n}^{t_{0}}(\lambda+i c) \sigma_{0}^{y} & =\frac{\Delta^{2}}{4}\left(\lambda-\frac{2 i Z_{n}}{\Delta}+i c\right)\left(\lambda+\frac{2 i Z_{n}}{\Delta}\right) \\
& =\frac{\Delta^{2}}{4}\left(\lambda-v_{n}\right)\left(\lambda-\bar{v}_{n}+i c\right) \quad \text { with } \quad v_{n}=-\frac{2 i Z_{n}}{\Delta} .
\end{aligned}
$$

Above and in the following, $\bar{z}$ stands for the complex conjugate of $z$.

It was observed by Izergin and Korepin ${ }^{6}$ that the zeroes of the quantum determinant define the values of the spectral parameter, where the Lax matrix has rank one. Namely, the Lax matrix (2.1) 
becomes a direct projector at the points $v_{n}, \bar{v}_{n}-i c$,

$$
\begin{aligned}
& {\left[L_{0 n}\left(v_{n}\right)\right]_{a b}=\alpha_{a}^{(+)}(n) \beta_{b}^{(+)}(n), \quad \text { with } \quad \alpha^{(+)}(n)=\left(\begin{array}{c}
\sqrt{c} \chi_{n}^{*} \\
2 i \rho_{Z_{n}}
\end{array}\right), \quad \beta^{(+)}(n)=\frac{1}{2}\left(\begin{array}{c}
\sqrt{c} \chi_{n} \\
-2 i \rho_{Z_{n}}
\end{array}\right),} \\
& {\left[L_{0 n}\left(\bar{v}_{n}-i c\right)\right]_{a b}=\alpha_{a}^{(-)}(n) \beta_{b}^{(-)}(n), \quad \text { with } \quad \alpha^{(-)}(n)=\left(\begin{array}{c}
-2 i \rho_{Z_{n}-\Delta c / 4} \\
\sqrt{c} \chi_{n}
\end{array}\right)} \\
& \beta^{(-)}(n)=\frac{1}{2}\left(\begin{array}{c}
2 i \rho_{Z_{n}-\Delta c / 4} \\
\sqrt{c} \chi_{n}^{*}
\end{array}\right) \text {. }
\end{aligned}
$$

It is a reverse projector at the points $v_{n}+i c, \bar{v}_{n}$,

$$
\begin{gathered}
{\left[L_{0 n}\left(v_{n}+i c\right)\right]_{a b}=\delta_{b}^{(+)}(n) \gamma_{a}^{(+)}(n), \quad \text { with } \quad \delta^{(+)}(n)=\frac{1}{2}\left(\begin{array}{c}
\sqrt{c} \chi_{n} \\
-2 i \rho_{Z_{n}-\Delta c / 4}
\end{array}\right),} \\
\gamma^{(+)}(n)=\left(\begin{array}{c}
\sqrt{c} \chi_{n}^{*} \\
2 i \rho_{Z_{n}-\Delta c / 4}
\end{array}\right), \\
{\left[L_{0 n}\left(\bar{v}_{n}\right)\right]_{a b}=\delta_{b}^{(-)}(n) \gamma_{a}^{(-)}(n), \quad \text { with } \quad \delta^{(-)}(n)=\left(\begin{array}{c}
2 i \rho_{Z_{n}} \\
\sqrt{c} \chi_{n}^{*}
\end{array}\right), \quad \gamma^{(-)}(n)=\frac{1}{2}\left(\begin{array}{c}
-2 i \rho_{Z_{n}} \\
\sqrt{c} \chi_{n}
\end{array}\right) .}
\end{gathered}
$$

\section{B. The lattice and the continuous models}

The Hamiltonian for the lattice model on an even number of sites $M$ is built out of the monodromy matrix

$$
T_{0 ; 1 \ldots M}(\lambda) \equiv T_{0}(\lambda)=L_{0 M}(\lambda) \ldots L_{01}(\lambda)=\left(\begin{array}{l}
A(\lambda) B(\lambda) \\
C(\lambda) D(\lambda)
\end{array}\right) \quad \text { with } \quad M \in 2 \mathbb{Z} .
$$

We have represented it as a $2 \times 2$ matrix on the auxiliary space $V_{0}$ whose entries are operators acting on the quantum space $\mathcal{H}=\otimes_{n=1}^{M} \mathcal{H}_{n}$. In the following, we set

$$
v \equiv v_{2 n-1}=v_{2 n}+i c=-\frac{2 i}{\Delta}+i \frac{c}{2} .
$$

The fact that Lax matrices become projectors (or reverse projectors) at $\lambda=v$ allows one to build the below local Hamiltonian out of the transfer matrix $\tau(\lambda)=\operatorname{tr}_{0}\left[T_{0}(\lambda)\right]$,

$$
\begin{aligned}
\tau^{-1}(v) \cdot \tau^{\prime}(v)=\sum_{k=1}^{M / 2}\left\{{ }^{t_{0}}\left[\beta^{(+)}(2 k+1)\right] L_{02 k}(v) L_{02 k-1}(v) \gamma^{(+)}(2 k-2)\right\}^{-1} \\
\cdot{ }^{t_{0}}\left[\beta^{(+)}(2 k+1)\right] \partial_{\lambda}\left[L_{02 k}(\lambda) L_{02 k-1}(\lambda)\right]_{\mid \lambda=v}^{\prime} \gamma^{(+)}(2 k-2),
\end{aligned}
$$

where ${ }^{t_{0}}$ refers to the operation of transposition of the vector $\beta^{(+)}(2 k+1)$. According to Izergin and Korepin, ${ }^{6}$ the above local Hamiltonians goes, in the continuum limit

$$
\Delta \rightarrow 0 \text { with } L=\Delta M \quad \text { fixed }
$$

to the Hamiltonian of the NLSM,

$$
\boldsymbol{H}_{\mathrm{NLS}}=\int_{0}^{L}\left\{\partial_{y} \Phi^{\dagger}(y) \partial_{y} \Phi(y)+c \Phi^{\dagger}(y) \Phi^{\dagger}(y) \Phi(y) \Phi(y)\right\} \mathrm{d} y .
$$

In (2.12) $\Phi$ and $\Phi^{\dagger}$ are canonical Bose fields subject to $L$ periodic boundary conditions. In such a continuous limit, the $k$ th site of the lattice model can be thought of as contributing to the "continuous 
coordinate" $x_{k}=k \Delta$. Then, the discreet fields $\chi_{n}$ are expected to be related to the canonical Bose fields $\Phi(x)$ as

$$
\chi_{n}=\int_{n \Delta}^{(n+1) \Delta} \Phi(x) \mathrm{d} x
$$

However, such an identification can only be given a formal sense in as much as, strictly speaking, the rhs does not have a precise mathematical meaning. On the other hand, the lhs has a sense in its own: the local operators $\chi_{n}$ and $\chi_{n}^{*}$ can be constructed explicitly, for instance as the harmonic oscillator creation/annihilation operators.

\section{The spectrum and eigenvectors}

The transfer matrix $\lambda \mapsto \tau(\lambda)$ is diagonalized by means of standard considerations of the algebraic Bethe Ansatz. One introduces the so-called pseudo-vacuum state $|0\rangle=|0\rangle_{1} \otimes \ldots|0\rangle_{M}$, where $|0\rangle_{n}$ is uniquely defined by the condition $\chi_{n}|0\rangle_{n}=0$ for all $n$. The commutation relations issuing from the Yang-Baxter equation (2.3),

$$
\begin{gathered}
A(\lambda) B(\mu)=\frac{\lambda-\mu+i c}{\lambda-\mu} B(\mu) A(\lambda)-\frac{i c}{\lambda-\mu} B(\lambda) A(\mu), \\
D(\lambda) B(\mu)=\frac{\lambda-\mu-i c}{\lambda-\mu} B(\mu) D(\lambda)+\frac{i c}{\lambda-\mu} B(\lambda) D(\mu),
\end{gathered}
$$

lead to the conclusion that the state

$$
\left|\psi\left(\left\{\lambda_{a}\right\}_{1}^{N}\right)\right\rangle=B\left(\lambda_{1}\right) \ldots B\left(\lambda_{N}\right)|0\rangle
$$

is an eigenstate of the transfer matrix $\tau(\lambda)$ associated with the eigenvalue

$$
\Lambda\left(\lambda \mid\{\lambda\}_{1}^{N}\right)=a(\lambda) \prod_{p=1}^{N} \frac{\lambda-\lambda_{p}+i c}{\lambda-\lambda_{p}}+d(\lambda) \prod_{p=1}^{N} \frac{\lambda-\lambda_{p}-i c}{\lambda-\lambda_{p}},
$$

where

$$
\begin{aligned}
& a(\lambda)=\left\{-i \frac{\lambda \Delta}{2}+1+\frac{c \Delta}{4}\right\}^{\frac{M}{2}} \cdot\left\{-i \frac{\lambda \Delta}{2}+1-\frac{c \Delta}{4}\right\}^{\frac{M}{2}} \text { and } \\
& d(\lambda)=\left\{i \frac{\lambda \Delta}{2}+1+\frac{c \Delta}{4}\right\}^{\frac{M}{2}} \cdot\left\{i \frac{\lambda \Delta}{2}+1-\frac{c \Delta}{4}\right\}^{\frac{M}{2}}
\end{aligned}
$$

provided that the parameters $\left\{\lambda_{a}\right\}_{1}^{N}$ solve the Bethe Ansatz equations (BAE),

$$
\frac{d\left(\lambda_{r}\right)}{a\left(\lambda_{r}\right)}=\prod_{\substack{p=1 \\ p \neq r}}^{N} \frac{\lambda_{r}-\lambda_{p}+i c}{\lambda_{r}-\lambda_{p}-i c} \quad, \quad r=1, \ldots, N .
$$

The solutions to (2.19) are real valued, satisfy to the so-called repulsion principle,

$$
\text { if } a \neq b, \quad \text { then } \quad \lambda_{a} \neq \lambda_{b},
$$

and are in a one-to-one correspondence with a certain subset (depending on $\Delta$ and $L$ for $\Delta M=L$ fixed) of the sets of all ordered integers $\ell_{1}<\cdots<\ell_{N}, \ell_{a} \in \underset{\widetilde{Z}}{\mathbb{Z}}$. More precisely, given any choice of integers $\ell_{1}<\cdots<\ell_{N}$, there exists a $\widetilde{\Delta}$ such that, for $\Delta<\widetilde{\Delta}$ (with $\Delta M=L$ fixed) there exists a 
unique solution to the below set of logarithmic Bethe equations

$$
\begin{aligned}
-i \ln \left(\frac{d\left(\mu_{\ell_{r}}\right)}{a\left(\mu_{\ell_{r}}\right)}\right)+\sum_{p=1}^{N} \theta\left(\mu_{\ell_{r}}-\mu_{\ell_{p}}\right) & =2 \pi\left(\ell_{r}-\frac{N+1}{2}\right), \quad r=1, \ldots, N \quad \text { with } \\
\theta(\lambda) & =i \ln \left(\frac{i c+\lambda}{i c-\lambda}\right) .
\end{aligned}
$$

Finally, using elementary properties of (2.21), it can be shown that, given a fixed product $\Delta M=L$ and any choice of integers $\ell_{1}<\cdots<\ell_{N}$, there exists a $\Delta_{0}>0$ such that the parameters $\mu_{\ell_{a}}$ $=\mu_{\ell_{a}}(\Delta)$ are continuous in $\Delta \in\left[0 ; \Delta_{0}\right]$.

In fact, the $\Delta \rightarrow 0, M \Delta=L$ limit of such a solution $\mu_{\ell_{a}}^{\mathrm{c}}=\lim _{\Delta \rightarrow 0} \mu_{\ell_{a}}(\Delta)$ gives rise to the set of parameters solving the logarithmic Bethe equations arising in the $N$ quasi-particle sector of the continuous model described by the Hamiltonian (2.12),

$$
L \mu_{\ell_{r}}^{\mathrm{c}}+\sum_{p=1}^{N} \theta\left(\mu_{\ell_{r}}^{\mathrm{c}}-\mu_{\ell_{p}}^{\mathrm{c}}\right)=2 \pi\left(\ell_{r}-\frac{N+1}{2}\right), \quad r=1, \ldots, N .
$$

Throughout this paper, we will always use the superscript c so as to indicate that $\left\{\mu_{\ell_{a}}^{\mathrm{c}}\right\}_{1}^{N}$ stands for the solution of the Bethe Ansatz equations for the continuous model. Likewise, the absence of such a superscript will indicate that one deals with the solution of the model at finite $\Delta$. We will omit the explicit writing of this $\Delta$ dependence.

It has been shown in Ref. 5 that the vectors $\left|\psi\left(\left\{\mu_{\ell_{a}}\right\}_{1}^{N}\right)\right\rangle$ converge, in some suitable sense, to the eigenfunctions,

$$
\left|\Psi\left(\left\{\mu_{\ell_{a}}^{\mathrm{c}}\right\}_{a=1}^{N}\right)\right\rangle=\int_{0}^{L} \varphi\left(x_{1}, \ldots, x_{N} \mid\left\{\mu_{\ell_{a}}^{\mathrm{c}}\right\}_{1}^{N}\right) \Phi^{\dagger}\left(x_{1}\right) \ldots \Phi^{\dagger}\left(x_{N}\right)|0\rangle \mathrm{d}^{N} x,
$$

of the continuous Hamiltonian (2.12) in the $N$ quasi-particle sector. The function $\varphi\left(x_{1}, \ldots, x_{N} \mid\left\{\lambda_{a}\right\}_{1}^{N}\right)$ can be constructed by means of the coordinate Bethe Ansatz ${ }^{14}$ and reads

$\varphi\left(x_{1}, \ldots, x_{N} \mid\left\{\lambda_{a}\right\}_{1}^{N}\right)=(-i \sqrt{c})^{N} \sum_{\sigma \in \mathfrak{S}_{N}} \prod_{a<b}^{N}\left\{\frac{\lambda_{\sigma(a)}-\lambda_{\sigma(b)}-i c \operatorname{sgn}\left(x_{a}-x_{b}\right)}{\lambda_{\sigma(a)}-\lambda_{\sigma(b)}}\right\} \cdot \prod_{a=1}^{N} \mathrm{e}^{i \lambda_{\sigma(a)} x_{a}} \mathrm{e}^{-i \lambda_{\sigma(a)} \frac{L}{2}}$

In (2.24), we made use of the following definition for the sign function:

$$
\operatorname{sgn}(x)=1 \text { for } x>0, \quad \operatorname{sgn}(x)=0 \quad \text { for } x=0, \quad \operatorname{sgn}(x)=-1 \text { for } x<0 .
$$

\section{Structure of the space of states}

The very setting of the algebraic Bethe Ansatz allows one to characterize the structure of the space of states by providing determinants representations for the norms ${ }^{11}$ and the scalar products between Bethe vectors. $^{21}$

Proposition 2.1 (Ref. 11 ): Let $\left\{\mu_{\ell_{a}}\right\}_{1}^{N+1}$ be any solution to the Bethe Ansatz equations (2.21), then the norm of the associated Bethe state admits the below determinant representation

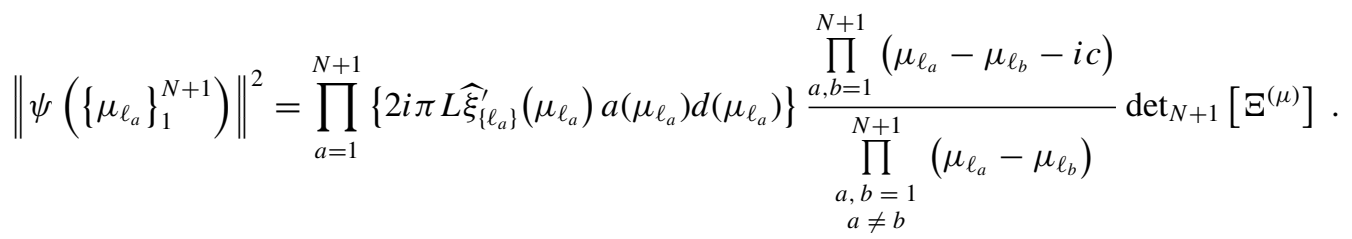


The entries of the matrix $\Xi^{(\mu)}$ read

$$
\begin{aligned}
\Xi_{j k}^{(\mu)}= & \delta_{j k}-\frac{K\left(\mu_{\ell_{a}}-\mu_{\ell_{b}}\right)}{2 \pi L \widehat{\xi}_{\left\{\ell_{a}\right\}}^{\prime}\left(\mu_{\ell_{b}}\right)} \text { with } \widehat{\xi}_{\left\{\ell_{a}\right\}}(\omega)=-\frac{i}{2 \pi L} \ln \left(\frac{d(\omega)}{a(\omega)}\right) \\
& +\frac{1}{2 \pi L} \sum_{p=1}^{N+1} \theta\left(\omega-\mu_{\ell_{p}}\right)+\frac{N+2}{2 L},
\end{aligned}
$$

and we have agreed upon $K(\lambda)=\theta^{\prime}(\lambda)$.

Theorem 2.1 (Ref. 21): Let $\left\{\mu_{\ell_{a}}\right\}_{1}^{N+1}$ be a solution to the logarithmic Bethe equations (2.21) and $\left\{\lambda_{a}\right\}_{1}^{N+1}$ a generic set of parameters. Then, the below scalar product reads

$$
\left\langle\psi\left(\left\{\mu_{\ell_{a}}\right\}_{1}^{N+1}\right) \mid \psi\left(\left\{\lambda_{a}\right\}_{1}^{N+1}\right)\right\rangle=\frac{\prod_{a=1}^{N+1} d\left(\mu_{\ell_{a}}\right)}{\prod_{a>b}^{N+1}\left(\mu_{\ell_{a}}-\mu_{\ell_{b}}\right)\left(\lambda_{b}-\lambda_{a}\right)} \operatorname{det}_{N+1}\left[\Omega\left(\left\{\mu_{\ell_{a}}\right\},\left\{\lambda_{a}\right\}\right)\right]
$$

where

$$
\left[\Omega\left(\left\{\mu_{a}\right\},\left\{\lambda_{a}\right\}\right)\right]_{j k}=a\left(\lambda_{k}\right) t\left(\mu_{j}, \lambda_{k}\right) \prod_{a=1}^{N+1}\left(\mu_{a}-\lambda_{k}-i c\right)-d\left(\lambda_{k}\right) t\left(\lambda_{k}, \mu_{j}\right) \prod_{a=1}^{N+1}\left(\mu_{a}-\lambda_{k}+i c\right)
$$

and

$$
t(\lambda, \mu)=\frac{-i c}{(\lambda-\mu)(\lambda-\mu-i c)}
$$

It was found by Oota ${ }^{17}$ that the reduction of the Lax matrix to projectors at zeroes of the quantum determinant that allows one to build local Hamiltonians from the transfer matrix can also be used to reconstruct certain local operators of the theory. In particular, one has the identity

$$
\tau^{-1}(v) \cdot B(v)=\left\{\sum_{r=1}^{2} \gamma_{r}^{(+)}(M) \beta_{r}^{(+)}(1)\right\}^{-1} \cdot \gamma_{1}^{(+)}(M) \beta_{2}^{(+)}(1) .
$$

Using the explicit formulas for $\gamma^{(+)}(k) \beta^{(+)}(k)$, one gets

$$
\sum_{r=1}^{2} \gamma_{r}^{(+)}(M) \beta_{r}^{(+)}(1)=\frac{c}{2} \chi_{M}^{*} \chi_{1}+2 \rho_{Z_{M}-\frac{\Delta c}{4}} \rho_{Z_{1}} \quad \text { and } \quad \gamma_{1}^{(+)}(M) \beta_{2}^{(+)}(1)=-i \sqrt{c} \chi_{M}^{*} \rho_{Z_{1}} .
$$

Thus, at least formally, one expects the below reconstruction formula for operators in the continuous model to hold

$$
\tau^{-1}(v) \cdot B(v)=-\frac{i \sqrt{c}}{2} \Delta \Phi^{\dagger}(0)+\mathrm{O}\left(\Delta^{2}\right)
$$

\section{FORM FACTORS OF THE CONJUGATED FIELD OPERATOR}

The formal identification (2.33) of products of entries of the monodromy matrix with operators in the continuous model can be made rigorous. This is one of the main results of this paper. It allows one to provide the missing steps in the passage from determinant representations for certain local operators in the lattice model obtained through the solution of the inverse problem ${ }^{17}$ to those for the form factors of the local operators in the continuous case. The proof of this theorem is postponed to Appendix. There, we also prove a similar result for the determinant representations of scalar products for the continuous model. 
Theorem 3.1: Let $\left\{\lambda_{\ell_{a}}\right\}_{1}^{N}$ be a solution of the logarithmic Bethe equations (2.21) in the $N$ particle sector and $\left\{\mu_{a}\right\}_{1}^{N}$ a set of generic, pairwise distinct complex numbers. Then the below scalar product in the lattice model converges, in the $\Delta \rightarrow 0$ limit, to the scalar product in the continuous model

$$
\left\langle\psi\left(\left\{\mu_{a}\right\}_{1}^{N}\right) \mid \psi\left(\left\{\lambda_{\ell_{a}}\right\}_{1}^{N}\right)\right\rangle \underset{\Delta \rightarrow 0}{\longrightarrow} \int_{0}^{L} \frac{\mathrm{d}^{N} x}{N !} \overline{\varphi\left(x_{1}, \ldots, x_{N} \mid\left\{\mu_{a}\right\}_{1}^{N}\right)} \varphi\left(x_{1}, \ldots, x_{N} \mid\left\{\lambda_{\ell_{a}}^{\mathrm{c}}\right\}_{1}^{N}\right) .
$$

As a consequence, one has the below determinant representation for the scalar products in the continuous model

$$
\frac{\prod_{a=1}^{N} d\left(\lambda_{\ell_{a}}^{\mathrm{c}}\right)}{\prod_{a>b}^{N}\left(\lambda_{\ell_{a}}^{\mathrm{c}}-\lambda_{\ell_{b}}^{\mathrm{c}}\right)\left(\mu_{b}-\mu_{a}\right)} \operatorname{det}_{N}\left[\Omega\left(\left\{\lambda_{\ell_{a}}^{\mathrm{c}}\right\}_{1}^{N},\left\{\mu_{a}\right\}_{1}^{N}\right)\right] .
$$

Theorem 3.2: Let $\left\{\mu_{\ell_{a}}\right\}_{1}^{N+1}$ and $\left\{\lambda_{r_{a}}\right\}_{1}^{N}$ be any two solution of the logarithmic Bethe equations (2.21) in the $N+1$ and $N$ particle sectors, respectively. Then, the expectation value,

$$
F_{\Phi^{\dagger}}^{(\Delta)}\left(\left\{\mu_{\ell_{a}}\right\}_{1}^{N+1} ;\left\{\lambda_{r_{a}}\right\}_{1}^{N}\right)=\frac{2 i}{\Delta \sqrt{c}} \cdot\left\langle\psi\left(\left\{\mu_{\ell_{a}}\right\}_{1}^{N+1}\right)\left|\tau^{-1}(\nu) B(v)\right| \psi\left(\left\{\lambda_{r_{a}}\right\}_{1}^{N}\right)\right\rangle,
$$

converges to the below form factor of the field operator in the continuous model

$$
F_{\Phi^{\dagger}}\left(\left\{\mu_{\ell_{a}}^{\mathrm{c}}\right\}_{1}^{N+1} ;\left\{\lambda_{r_{a}}^{\mathrm{c}}\right\}_{1}^{N}\right)=\int_{0}^{L} \frac{\mathrm{d}^{N} x}{N !} \overline{\varphi\left(0, x_{1}, \ldots, x_{N} \mid\left\{\mu_{\ell_{a}}^{\mathrm{c}}\right\}_{1}^{N+1}\right)} \cdot \varphi\left(x_{1}, \ldots, x_{N} \mid\left\{\lambda_{r_{a}}^{\mathrm{c}}\right\}_{1}^{N}\right) .
$$

The latter admits the below determinant representation

$$
\begin{aligned}
& F_{\Phi^{\ddagger}}\left(\left\{\mu_{\ell_{a}}^{\mathrm{c}}\right\}_{1}^{N+1} ;\left\{\lambda_{r_{a}}^{\mathrm{c}}\right\}_{1}^{N}\right)= i \sqrt{c} \prod_{a=1}^{N+1} \mathrm{e}^{\frac{i L}{2} \mu_{\ell_{a}}^{\mathrm{c}}} \cdot \prod_{k=1}^{N}\left\{\mathrm{e}^{-\frac{i L}{2} \lambda_{r_{k}}^{\mathrm{c}}}\left[1-\mathrm{e}^{-2 i \pi \widehat{F}^{\mathrm{c}}\left(\lambda_{r_{k}}^{\mathrm{c}}\right)}\right] \prod_{b=1}^{N+1} \frac{\mu_{\ell_{b}}^{\mathrm{c}}-\lambda_{r_{k}}^{\mathrm{c}}-i c}{\mu_{\ell_{b}}^{\mathrm{c}}-\lambda_{r_{k}}^{\mathrm{c}}}\right\} \operatorname{det}_{N} \\
& \times\left[\delta_{j k}+U_{j k}\right], \\
& U_{j k}=-i \prod_{a=1}^{N+1} \frac{\lambda_{r_{j}}^{\mathrm{c}}-\mu_{\ell_{a}}^{\mathrm{c}}}{\lambda_{r_{j}}^{\mathrm{c}}-\mu_{\ell_{a}}^{\mathrm{c}}+i c} \cdot \frac{\prod_{a=1}^{N}\left(\lambda_{r_{j}}^{\mathrm{c}}-\lambda_{r_{a}}^{\mathrm{c}}+i c\right)}{\prod_{\substack{a=1 \\
\neq j}}^{N}\left(\lambda_{r_{j}}^{\mathrm{c}}-\lambda_{r_{a}}^{\mathrm{c}}\right)} \cdot \frac{K\left(\lambda_{r_{j}}^{\mathrm{c}}-\lambda_{r_{k}}^{\mathrm{c}}\right)}{\mathrm{e}^{-2 i \pi \widehat{F}^{\mathrm{c}}\left(\lambda_{r_{j}}^{\mathrm{c}}\right)}-1} .
\end{aligned}
$$

Here, we made use of the so-called the discrete shift function $\widehat{F}^{\mathrm{c}}$ for the continuous model

$$
\mathrm{e}^{-2 i \pi \widehat{F}^{\mathrm{c}}(\omega)}=\prod_{a=1}^{N+1} \frac{\mu_{\ell_{a}}^{\mathrm{c}}-\omega+i c}{\mu_{\ell_{a}}^{\mathrm{c}}-\omega-i c} \cdot \prod_{a=1}^{N} \frac{\lambda_{r_{a}}^{\mathrm{c}}-\omega-i c}{\lambda_{r_{a}}^{\mathrm{c}}-\omega+i c} .
$$

\section{A. Determinant representation in the lattice model}

Determinant representations for the form factors of the conjugated field operator in the NLSM have been obtained in Ref. 10 through the use of the two-site models and in Ref. 17 with the help of the inverse problem previously discussed. These results all relied on the hypothesis of the convergence of the lattice discretization to the continuous model has been proven in Theorem 3.2 above. Actually, we have provided a slightly different (in respect to the aforecited Refs. 10 and 17) determinant representation for $F_{\Phi^{\dagger}}\left(\left\{\mu_{\ell_{a}}^{\mathrm{c}}\right\}_{1}^{N+1} ;\left\{\lambda_{r_{a}}^{\mathrm{c}}\right\}_{1}^{N}\right)$. The equivalence of our representation with 
the previous one can, in principle, be checked with the help of determinant identities analogous to those established in Refs. 7 and 8. We now derive a determinant representation for $F_{\Phi^{\dagger}}^{(\Delta)}$ defined in (3.3). This provides a slightly different representation in respect to the one obtained by Oota. ${ }^{17}$

Proposition 3.1: The discrete approximation $F_{\Phi^{\dagger}}^{(\Delta)}\left(\left\{\mu_{\ell_{a}}\right\}_{1}^{N+1} ;\left\{\lambda_{r_{a}}\right\}_{1}^{N}\right)$ defined in (3.3) admits the determinant representation

$$
\begin{gathered}
F_{\Phi^{\dagger}}^{(\Delta)}\left(\left\{\mu_{\ell_{a}}\right\}_{1}^{N+1} ;\left\{\lambda_{a}\right\}_{1}^{N}\right)=\frac{-2 \sqrt{c}}{\Delta} \prod_{a=1}^{N+1} \frac{v-\mu_{\ell_{a}}}{v-\mu_{\ell_{a}}-i c} \frac{\prod_{a=1}^{N}\left(\lambda_{r_{a}}-v+i c\right)}{\prod_{a=1}^{N+1}\left(\mu_{\ell_{a}}-v\right)} \prod_{a=1}^{N+1} \prod_{b=1}^{N} \frac{1}{\mu_{\ell_{a}}-\lambda_{r_{b}}} \\
\prod_{a=1}^{N+1} d\left(\mu_{\ell_{a}}\right) \prod_{k=1}^{N}\left\{a\left(\lambda_{r_{k}}\right)\left[1-\mathrm{e}^{-2 i \pi \widehat{F}\left(\lambda_{r_{k}}\right)}\right] \prod_{b=1}^{N+1}\left(\mu_{\ell_{a}}-\lambda_{r_{k}}-i c\right)\right\} \cdot \operatorname{det}_{N}\left[\delta_{j k}+U_{j k}^{(\Delta)}\right], \\
U_{j k}^{(\Delta)}=-i \prod_{a=1}^{N+1} \frac{\lambda_{r_{j}}-\mu_{\ell_{a}}}{\lambda_{r_{j}}-\mu_{\ell_{a}}+i c} \cdot \frac{\prod_{a=1}^{N}\left(\lambda_{r_{j}}-\lambda_{r_{a}}+i c\right)}{\prod_{\substack{a=1 \\
\neq j}}^{N}\left(\lambda_{r_{j}}-\lambda_{r_{a}}\right)} \cdot \frac{K\left(\lambda_{r_{j}}, \lambda_{r_{k}} \mid v\right)}{\mathrm{e}^{-2 i \pi \hat{F}\left(\lambda_{r_{j}}\right)}-1},
\end{gathered}
$$

and, recalling that $K(\lambda)=\theta^{\prime}(\lambda)$ with $\theta(\lambda)$ given in (2.21) and $v$ in (2.10),

$$
K\left(\omega, \omega^{\prime} \mid v\right)=\frac{v-\omega-i c}{v-\omega}\left\{K\left(\omega-\omega^{\prime}\right)-i\left(1-\frac{v-\omega^{\prime}+i c}{v-\omega^{\prime}-i c}\right)\left(\frac{1}{\omega-\omega^{\prime}+i c}-\frac{1}{\omega-v+i c}\right)\right\} .
$$

Also, above, we made use of the so-called the discrete shift function $\widehat{F}^{\mathrm{c}}$ for the continuous model

$$
\mathrm{e}^{-2 i \pi \widehat{F}(\omega)}=\prod_{a=1}^{N+1} \frac{\mu_{\ell_{a}}-\omega+i c}{\mu_{\ell_{a}}-\omega-i c} \cdot \prod_{a=1}^{N} \frac{\lambda_{r_{a}}-\omega-i c}{\lambda_{r_{a}}-\omega+i c}
$$

Proof: Using that $\left|\psi\left(\left\{\mu_{\ell_{a}}\right\}_{1}^{N+1}\right)\right\rangle$ is an eigenstate of $\tau^{-1}(\lambda)$ for any $\lambda$, it readily follows that $F_{\Phi^{\dagger}}^{(\Delta)}\left(\left\{\mu_{\ell_{a}}\right\}_{1}^{N+1} ;\left\{\lambda_{r_{a}}\right\}_{1}^{N}\right)=\frac{2 i}{\Delta \sqrt{c}}[d(v)]^{-1} \prod_{a=1}^{N+1} \frac{v-\mu_{\ell_{a}}}{v-\mu_{\ell_{a}}-i c} \cdot\left\langle\psi\left(\left\{\mu_{\ell_{a}}\right\}_{1}^{N+1}\right) \mid \psi\left(\left\{\lambda_{r_{a}}\right\}_{1}^{N+1}\right)\right\rangle$.

In the scalar product formula, we agree upon $\lambda_{r_{N+1}} \equiv v$.

Using techniques proposed in Refs. 7 and 8, it is possible to factor out a Cauchy determinant from the determinant of $\Omega$. This leads to the representation

$$
\operatorname{det}_{N+1}\left[\Omega\left(\left\{\mu_{\ell_{a}}\right\},\left\{\lambda_{r_{a}}\right\}\right)\right]=\operatorname{det}_{N+1}\left[\frac{1}{\mu_{\ell_{a}}-\lambda_{r_{b}}}\right] \cdot \operatorname{det}_{N+1}[S] .
$$

The matrix $S$ takes the form

$$
S_{j k}=\delta_{j k} \mathcal{Y}\left(\lambda_{r_{k}} \mid\left\{\mu_{\ell_{a}}\right\}_{1}^{N+1}\right)+\left.\frac{\prod_{\substack{a=1 \\ a \neq 1 \\ a \neq j}}^{N+1}\left(\lambda_{r_{j}}-\mu_{\ell_{a}}\right)}{\prod_{\substack{a \\ \text { a }}}^{N+1}\left(\lambda_{r_{j}}-\lambda_{r_{a}}\right)} \cdot \frac{\partial}{\partial y_{j}} \mathcal{Y}\left(\lambda_{k} \mid\left\{y_{a}\right\}\right)\right|_{\left\{y_{a}\right\}=\left\{\lambda_{r_{a}}\right\}}
$$

for $k \in \llbracket 1 ; N+1 \rrbracket, j \in \llbracket 1 ; N \rrbracket$ and $S_{N+1 k}=\mathcal{Y}\left(\lambda_{r_{k}} \mid\left\{\lambda_{r_{a}}\right\}\right)$. 
Above, we have set

$$
\mathcal{Y}\left(\lambda \mid\{\tau\}_{1}^{N+1}\right)=a(\lambda) \prod_{k=1}^{N+1}\left(\tau_{k}-\lambda-i c\right)+d(\lambda) \prod_{k=1}^{N+1}\left(\tau_{k}-\lambda+i c\right) .
$$

One can reduce the dimensionality of $\operatorname{det}_{N+1}[S]$ by 1 thanks to the below linear combination of columns

$$
C_{k} \leftarrow C_{k}-\frac{\mathcal{Y}\left(\lambda_{r_{k}} \mid\left\{\lambda_{r_{a}}\right\}_{1}^{N+1}\right)}{\mathcal{Y}\left(v \mid\left\{\lambda_{r_{a}}\right\}_{1}^{N+1}\right)} \cdot C_{N+1} .
$$

Then, using explicitly that $\lambda_{r_{N+1}}=v$, one gets

$$
\operatorname{det}_{N+1}[S]=\mathcal{Y}\left(v \mid\left\{\lambda_{r_{a}}\right\}_{1}^{N+1}\right) \cdot \operatorname{det}_{N}\left[S_{j k}-S_{j N+1} \frac{\mathcal{Y}\left(\lambda_{r_{k}} \mid\left\{\lambda_{r_{a}}\right\}_{1}^{N+1}\right)}{\mathcal{Y}\left(v \mid\left\{\lambda_{r_{a}}\right\}_{1}^{N+1}\right)}\right] .
$$

The functions $\mathcal{Y}\left(\lambda_{r_{k}} \mid\left\{\mu_{\ell_{a}}\right\}_{1}^{N+1}\right)$ can be recast in terms of the shift function $\widehat{F}\left(\lambda_{r_{k}}\right)$ given in (3.11),

$$
\mathcal{Y}\left(\lambda_{r_{k}} \mid\left\{\mu_{\ell_{a}}\right\}_{1}^{N+1}\right)=a\left(\lambda_{k}\right) \prod_{a=1}^{N+1}\left(\mu_{\ell_{a}}-\lambda_{r_{k}}-i c\right) \cdot\left\{1-\mathrm{e}^{-2 i \pi \widehat{F}\left(\lambda_{r_{k}}\right)}\right\} .
$$

To obtain (3.18), we have used that $\left\{\lambda_{r_{k}}\right\}_{k=1}^{N}$ is the solution of the $N$-particle BAE. Then, computing explicitly the difference in the determinant and factoring out the $\mathcal{Y}$ functions, we get that

$$
\begin{aligned}
\operatorname{det}_{N+1}\left[\Omega\left(\left\{\mu_{\ell_{a}}\right\},\left\{\lambda_{r_{a}}\right\}\right)\right]= & \mathcal{Y}\left(v \mid\left\{\lambda_{r_{a}}\right\}_{1}^{N+1}\right) \cdot \prod_{k=1}^{N} \mathcal{Y}\left(\lambda_{r_{k}} \mid\left\{\mu_{\ell_{a}}\right\}_{1}^{N+1}\right) \\
& \cdot \operatorname{det}_{N+1}\left[\frac{1}{\mu_{\ell_{a}}-\lambda_{r_{a}}}\right] \operatorname{det}_{N}\left[\delta_{j k}+U_{j k}^{(\Delta)}\right] .
\end{aligned}
$$

It only remains to put all the formulas together.

\section{LARGE VOLUME BEHAVIOR OF THE FORM FACTORS OF CONJUGATED FIELDS}

In this subsection, we provide formulas for the large volume limit of the form factors $F_{\Phi^{\ddagger}}$ for a specific class of excited states. Namely, we assume that the state described by $\left\{\mu_{\ell_{a}}^{\mathrm{c}}\right\}_{1}^{N+1}$ corresponds to an $n$-particle/hole excitation above the $N+1$-quasi particle ground state, whereas the state $\left\{\lambda_{r_{a}}^{\mathrm{c}}\right\}_{1}^{N}$ stands for the ground state (i.e., $r_{a}=a$ for $a=1, \ldots, N$ ) in the $N$-quasi particle sector. The methods for carrying out such computations have been developed in Refs. 7,9, and 22.

\section{A. Rudiments of the thermodynamic limit in the NLSM}

Given the set of Bethe roots $\left\{\lambda_{a}^{c}\right\}$ for the ground state in the $N$ quasi-particle sector, one builds their counting function as

$$
\widehat{\xi}(\omega) \equiv \widehat{\xi}\left(\omega \mid\left\{\lambda_{a}^{\mathrm{c}}\right\}_{1}^{N}\right)=\frac{\omega}{2 \pi}+\frac{1}{2 \pi L} \sum_{a=1}^{N} \theta\left(\omega-\lambda_{a}^{\mathrm{c}}\right)+\frac{N+1}{2 L}, \text { i.e., } \widehat{\xi}\left(\lambda_{a}^{\mathrm{c}}\right)=\frac{a}{L} .
$$

The latter has the below behavior in the thermodynamic limit of the model (i.e., $N, L \rightarrow+\infty$ with $N / L \rightarrow D)$,

$\widehat{\xi}(\omega)=\xi(\omega)+\mathrm{O}\left(L^{-1}\right)$, where $\quad \xi(\omega)=\frac{p(\omega)}{2 \pi}+\frac{D}{2}$ with $p(\lambda)-\int_{-q}^{q} \theta(\lambda-\mu) p^{\prime}(\mu) \frac{\mathrm{d} \mu}{2 \pi}=\lambda$. 
The parameter $q$ corresponds to the right end of the Fermi interval $[-q ; q]$ on which the ground state's Bethe roots condensate in the thermodynamic limit. It is defined as the unique solution to $p(q)=\pi D$.

Recall that any solution $\left\{\mu_{\ell_{a}}^{\mathrm{c}}\right\}_{1}^{N+1}$ of the Bethe equations in the $N+1$ quasi-particle sector is uniquely determined by the choice of $N+1$ integers $\ell_{1}<\cdots<\ell_{N+1}$. It is convenient to parameterize the integers $\ell_{j}$ in terms of particle-hole excitations above the $N+1$ quasi-particle ground state

$$
\ell_{j}=j \quad \text { for } j \in \mathbb{I} 1 ; N+1 \rrbracket \backslash h_{1}, \ldots, h_{n} \quad \text { and } \quad \ell_{h_{a}}=p_{a} \quad \text { for } a=1, \ldots, n \text {. }
$$

The integers $p_{a}$ and $h_{a}$ are such that $p_{a} \notin \llbracket 1 ; N+1 \rrbracket \equiv\{1, \ldots, N+1\}$ and $h_{a} \in \llbracket 1 ; N+1 \rrbracket$.

One can actually associate a counting function to any solution $\left\{\mu_{\ell_{a}}^{\mathrm{c}}\right\}_{1}^{N+1}$ by

$$
\widehat{\xi}_{\left\{\ell_{a}\right\}}(\omega) \equiv \widehat{\xi}_{\left\{\ell_{a}\right\}}\left(\omega \mid\left\{\mu_{\ell_{a}}^{\mathrm{c}}\right\}_{1}^{N+1}\right)=\frac{\omega}{2 \pi}+\frac{1}{2 \pi L} \sum_{a=1}^{N+1} \theta\left(\omega-\mu_{\ell_{a}}^{\mathrm{c}}\right)+\frac{N+2}{2 L} .
$$

By construction, it is such that $\widehat{\xi}_{\left\{\ell_{a}\right\}}\left(\mu_{\ell_{a}}^{\mathrm{c}}\right)=\ell_{a} / L$, for $a=1, \ldots, N+1$. Actually, $\widehat{\xi}_{\left\{\ell_{a}\right\}}(\omega)$ defines a set of background parameters $\left\{\widehat{\mu}_{a}\right\}, a \in \mathbb{Z}$, as the unique solutions to $\widehat{\xi}_{\left\{\ell_{a}\right\}}\left(\widehat{\mu}_{a}\right)=a / L$. The latter allows one to define the rapidities $\widehat{\mu}_{p_{a}}$, respectively, $\widehat{\mu}_{h_{a}}$, of the particles, respectively, holes, entering in the description of $\left\{\mu_{\ell_{a}}^{\mathrm{c}}\right\}_{1}^{N+1}$.

It can be shown that the shift function

$$
\widehat{F}^{\mathrm{c}}(\omega)=L\left[\widehat{\xi}(\omega)-\widehat{\xi}_{\left\{\ell_{a}\right\}}(\omega)\right]
$$

has a well-defined thermodynamic limit

$$
F(\lambda) \equiv F\left(\lambda \mid\left\{\mu_{p_{a}}\right\}\left\{\mu_{h_{a}}\right\}\right)=-Z(\lambda) / 2-\phi(\lambda, q)-\sum_{a=1}^{n}\left[\phi\left(\lambda, \mu_{p_{a}}\right)-\phi\left(\lambda, \mu_{h_{a}}\right)\right],
$$

where the dressed phase $\phi(\lambda, \mu)$ and the dressed charge $Z(\lambda)$ solve the linear integral equations

$$
\phi(\lambda, \mu)-\int_{-q}^{q} K(\lambda-\tau) \phi(\tau, \mu) \frac{\mathrm{d} \tau}{2 \pi}=\frac{1}{2 \pi} \theta(\lambda-\mu) \quad \text { and } \quad Z(\lambda)-\int_{-q}^{q} K(\lambda-\tau) Z(\tau) \frac{\mathrm{d} \tau}{2 \pi}=1 .
$$

This thermodynamic limit of the shift function depends on the particles' $\left\{\mu_{p_{a}}\right\}$ and holes' $\left\{\mu_{h_{a}}\right\}$ positions in the thermodynamic limit. These are defined as the solutions to

$$
\xi\left(\mu_{p_{a}}\right)=p_{a} / L \quad \text { and } \quad \xi\left(\mu_{h_{a}}\right)=h_{a} / L
$$

We remind that the above shift function measures the spacing between the ground state roots $\lambda_{a}$ and the background parameters $\widehat{\mu}_{a}$ defined by $\widehat{\xi}_{\left\{\ell_{a}\right\}}: \widehat{\mu}_{a}-\lambda_{a}=F\left(\lambda_{a}\right) \cdot\left[L \xi^{\prime}\left(\lambda_{a}\right)\right]^{-1}\left(1+\mathrm{O}\left(L^{-1}\right)\right)$.

\section{B. Thermodynamic limit of form factors}

By applying Propositions 2.1 and 3.1, it readily follows that the normalized modulus squared of the form factor of the conjugated field admits the factorization

$$
\frac{\left.\| \Psi \Psi\left(\left\{\mu_{\ell_{a}}^{\mathrm{c}}\right\}_{1}^{N+1}\right)\left|\Phi^{\dagger}(0)\right| \Psi\left(\left\{\lambda_{a}^{\mathrm{c}}\right\}_{1}^{N}\right)\right\rangle\left.\right|^{2}}{\left\|\Psi\left(\left\{\mu_{\ell_{a}}^{\mathrm{c}}\right\}_{1}^{N+1}\right)\right\|^{2}\left\|\Psi\left(\left\{\lambda_{a}^{\mathrm{c}}\right\}_{1}^{N}\right)\right\|^{2}}=\widehat{D}_{N}\left(\left\{\mu_{\ell_{a}}^{\mathrm{c}}\right\}_{1}^{N+1} ;\left\{\lambda_{a}^{\mathrm{c}}\right\}_{1}^{N}\right) \widehat{\mathcal{G}}_{N}\left(\left\{\mu_{\ell_{a}}^{\mathrm{c}}\right\}_{1}^{N+1} ;\left\{\lambda_{a}^{\mathrm{c}}\right\}_{1}^{N}\right),
$$

into the products of its so-called smooth part $\widehat{\mathcal{G}}_{N}$ and discreet part $\widehat{D}_{N}$. 
The smooth part reads

$$
\begin{aligned}
\widehat{\mathcal{G}}_{N}\left(\left\{\mu_{\ell_{a}}^{\mathrm{c}}\right\}_{1}^{N+1} ;\left\{\lambda_{a}^{\mathrm{c}}\right\}_{1}^{N}\right)= & \mathcal{W}_{N}\left(\begin{array}{c}
\left\{\mu_{\ell_{a}}^{\mathrm{c}}\right\}_{1}^{N} \\
\left\{\lambda_{a}^{\mathrm{c}}\right\}_{1}^{N}
\end{array}\right) \prod_{a=1}^{N}\left|\frac{\lambda_{a}^{\mathrm{c}}-\mu_{\ell_{N+1}}^{\mathrm{c}}-i c}{\mu_{\ell_{a}}^{\mathrm{c}}-\mu_{\ell_{N+1}}^{\mathrm{c}}-i c}\right|^{2} \\
& \frac{\operatorname{det}_{N}\left[\delta_{j k}+U_{j k}\right] \overline{\operatorname{det}_{N}\left[\delta_{j k}+U_{j k}\right]}}{\operatorname{det}_{N+1}\left[\Xi^{(\mu)}\right] \cdot \operatorname{det}_{N}\left[\Xi^{(\lambda)}\right]} .
\end{aligned}
$$

There

$$
\mathcal{W}_{N}\left(\begin{array}{c}
\left\{z_{a}\right\}_{1}^{N} \\
\left\{w_{a}\right\}_{1}^{N}
\end{array}\right)=\prod_{a, b=1}^{N} \frac{\left(z_{a}-w_{b}-i c\right)\left(w_{a}-z_{b}-i c\right)}{\left(z_{a}-z_{b}-i c\right)\left(w_{a}-w_{b}-i c\right)} .
$$

The discrete part takes the form

$$
\begin{aligned}
\widehat{D}_{N}\left(\left\{\mu_{\ell_{a}}^{\mathrm{c}}\right\}_{1}^{N+1} ;\left\{\lambda_{a}^{\mathrm{c}}\right\}_{1}^{N}\right)= & \frac{\prod_{k=1}^{N}\left\{4 \sin ^{2}\left[\pi F_{\left\{\ell_{a}\right\}}\left(\lambda_{k}^{\mathrm{c}}\right)\right]\right\}}{\prod_{a=1}^{N+1}\left\{2 \pi L \widehat{\xi}_{\left\{\ell_{a}\right\}}^{\prime}\left(\mu_{\ell_{a}}^{\mathrm{c}}\right)\right\} \prod_{a=1}^{N}\left\{2 \pi L \widehat{\xi}^{\prime}\left(\lambda_{a}^{\mathrm{c}}\right)\right\}} \cdot \prod_{a=1}^{N}\left(\frac{\mu_{\ell_{a}}^{\mathrm{c}}-\mu_{\ell_{N+1}}^{\mathrm{c}}}{\lambda_{a}^{\mathrm{c}}-\mu_{\ell_{N+1}}^{\mathrm{c}}}\right)^{2} \\
& \cdot \operatorname{det}_{N}^{2}\left[\frac{1}{\mu_{\ell_{a}}^{\mathrm{c}}-\lambda_{b}^{\mathrm{c}}}\right]
\end{aligned}
$$

In the remainder of this subsection, we discuss the large- $L$ behavior of these two quantities.

\section{The smooth part}

$\widehat{\mathcal{G}}_{N}$ is called the smooth part as its thermodynamic limit $\mathcal{G}_{n}$ only depends on the value of the rapidities of the particles $\left\{\mu_{p_{a}}\right\}_{1}^{n}$ and holes $\left\{\mu_{h_{a}}\right\}_{1}^{n}$ entering in the description of the thermodynamic limit of the excited state. We recall that these are defined as in (4.8). The function $\mathcal{G}_{n}$ can be readily expressed ${ }^{7}$ in terms of the thermodynamic limit $F$ (4.6) of the shift function associated with the excited state $\left\{\mu_{\ell_{a}}^{\mathrm{c}}\right\}_{1}^{N+1}$,

$$
\widehat{\mathcal{G}}_{N}\left(\left\{\mu_{\ell_{a}}^{\mathrm{c}}\right\}_{1}^{N+1} ;\left\{\lambda_{a}^{\mathrm{c}}\right\}_{1}^{N}\right)=\mathcal{G}_{n}\left(\begin{array}{l}
\left\{\mu_{p_{a}}\right\} \\
\left\{\mu_{h_{a}}\right\}
\end{array}\right)[F] \times\left(1+\mathrm{O}\left(L^{-1}\right)\right),
$$

with

$$
\begin{aligned}
& \mathcal{G}_{n}\left(\begin{array}{l}
\left\{\mu_{p_{a}}\right\} \\
\left\{\mu_{h_{a}}\right\}
\end{array}\right)[F]=\prod_{a=1}^{n} \prod_{\epsilon= \pm}\left\{\frac{\mu_{h_{a}}-q+\epsilon i c}{\mu_{p_{a}}-q+\epsilon i c} \frac{\mathrm{e}^{2 i \pi C[F]\left(\mu_{h_{a}}+\epsilon i c\right)}}{\mathrm{e}^{2 i \pi C[F]\left(\mu_{p a}+\epsilon i c\right)}}\right\} \cdot \frac{\mathrm{e}^{-2 i \pi \sum_{\epsilon= \pm} C[F](q+\epsilon i c)}}{\operatorname{det}^{2}[I-K / 2 \pi]} \mathrm{e}^{C_{0}[F]} \\
& \quad \times \mathcal{W}_{n}\left(\begin{array}{l}
\left\{\mu_{p_{a}}\right\} \\
\left\{\mu_{h_{a}}\right\}
\end{array}\right) \cdot \operatorname{det}_{\mathscr{C}_{q}}\left[I+U[F]\left(\left\{\mu_{p_{a}}\right\}_{1}^{n},\left\{\mu_{h_{a}}\right\}_{1}^{n}\right)\right] \operatorname{det}_{\mathscr{C}_{q}}\left[I+\bar{U}[F]\left(\left\{\mu_{p_{a}}\right\}_{1}^{n},\left\{\mu_{h_{a}}\right\}_{1}^{n}\right)\right] .
\end{aligned}
$$

There, $C[F]$ is the Cauchy transform on $[-q ; q]$ and $C_{0}[F]$ is given by a double integral

$$
C[F](\lambda)=\int_{-q}^{q} \frac{\mathrm{d} \mu}{2 i \pi} \frac{F(\mu)}{\mu-\lambda} \quad \text { and } \quad C_{0}[F]=-\int_{-q}^{q} \frac{F(\lambda) F(\mu)}{(\lambda-\mu-i c)^{2}} \mathrm{~d} \lambda \mathrm{d} \mu
$$

All determinants appearing in (4.14) are Fredholm determinants of integral operators of the type $I+A$. The integral operator $I-K / 2 \pi$ acts on $[-q ; q]$. The integral kernels $U$ and $\bar{U}$ are given by

$$
\begin{aligned}
U\left(\omega, \omega^{\prime}\right)[F]= & \frac{-1}{2 \pi} \frac{\omega-q}{\omega-q+i c} \prod_{a=1}^{n}\left\{\frac{\left(\omega-\mu_{p_{a}}\right)\left(\omega-\mu_{h_{a}}+i c\right)}{\left(\omega-\mu_{h_{a}}\right)\left(\omega-\mu_{p_{a}}+i c\right)}\right\} \\
& \cdot \mathrm{e}^{C[2 i \pi F](\omega)-C[2 i \pi F](\omega+i c)} \frac{K\left(\omega-\omega^{\prime}\right)}{\mathrm{e}^{-2 i \pi F(\omega)}-1}
\end{aligned}
$$


and

$$
\begin{aligned}
\bar{U}\left(\omega, \omega^{\prime}\right)[F]= & \frac{1}{2 \pi} \frac{\omega-q}{\omega-q-i c} \prod_{a=1}^{n}\left\{\frac{\left(\omega-\mu_{p_{a}}\right)\left(\omega-\mu_{h_{a}}-i c\right)}{\left(\omega-\mu_{h_{a}}\right)\left(\omega-\mu_{p_{a}}-i c\right)}\right\} \\
& \cdot \mathrm{e}^{C[2 i \pi F](\omega)-C[2 i \pi F](\omega-i c)} \frac{K\left(\omega-\omega^{\prime}\right)}{\mathrm{e}^{2 i \pi F(\omega)}-1} .
\end{aligned}
$$

Above, so as to lighten the notations, we have kept the dependence on the particles' and holes' rapidities implicit. The operators $I+U[F]$ and $I+\bar{U}[F]$ should de understood as acting on function defined on a counterclockwise contour $\mathscr{C}_{q}$ surrounding the interval $[-q ; q]$ but not any other singularity of the integrand. In particular, the poles at $\omega=\mu_{h_{a}}$ are located inside of $\mathscr{C}_{q}$, whereas the zeroes of $\lambda \mapsto \mathrm{e}^{-2 i \pi F(\lambda)}-1$ are located outside of the contour. In Sec. IV C below we provide a more precise definition of these determinants as, in principle, the existence of such a contour is not guaranteed for all possible choices of parameters $\left\{\mu_{p_{a}}\right\}$ and $\left\{\mu_{h_{a}}\right\}$.

\section{The discrete part}

The name discrete part originates in that the leading thermodynamic behavior of $\widehat{D}_{N}$ not only depends on the "macroscopic" rapidities $\left\{\mu_{p_{a}}\right\}$ and $\left\{\mu_{h_{a}}\right\}$ entering in the description of the excited state but also on the set of integers $\left\{p_{a}\right\}$ and $\left\{h_{a}\right\}$ characterizing the excited state. By using the techniques developed in Refs. 7,9, and 22, one readily shows that the leading in $L$ thermodynamic behavior of $\widehat{D}_{N}$ takes the form

$$
\widehat{D}_{N}\left(\left\{\mu_{\ell_{a}}^{\mathrm{c}}\right\}_{1}^{N+1} ;\left\{\lambda_{a}^{\mathrm{c}}\right\}_{1}^{N}\right)=D_{0}[F] \mathcal{R}_{N, n}\left(\begin{array}{c}
\left\{\mu_{p_{a}}\right\} ;\left\{p_{a}\right\} \\
\left\{\mu_{h_{a}}\right\} ;\left\{h_{a}\right\}
\end{array}\right)[F] \times\left(1+\mathrm{O}\left(\frac{\ln L}{L}\right)\right),
$$

where

$D_{0}[\nu]=\frac{2 q}{2 \pi} \cdot \frac{\left(\kappa_{-}[\nu]\right)^{\nu_{-}}}{\left(\kappa_{+}[\nu]\right)^{\nu_{+}+2}} \prod_{a=1}^{n}\left(\frac{\lambda_{N+1}-\mu_{p_{a}}}{\lambda_{N+1}-\mu_{h_{a}}}\right)^{2} \frac{G^{2}\left(1-v_{-}\right) G^{2}\left(2+v_{+}\right)}{(2 \pi)^{\nu_{+}-\nu_{-}} \cdot\left[2 q L \xi_{+}^{\prime}\right]^{\left(\nu_{+}+1\right)^{2}+v_{-}^{2}}} \cdot \mathrm{e}^{\frac{1}{2} \int_{-q}^{q} \frac{\nu^{\prime}(\lambda)(\mu)-\nu^{\prime}(\mu)(\lambda)}{\lambda-\mu} \mathrm{d} \lambda \mathrm{d} \mu}$.

The parameter $\lambda_{N+1}$ appearing above is defined as the unique solution to $L \xi\left(\lambda_{N+1}\right)-F\left(\lambda_{N+1}\right)$ $=N+1, G$ is the Barnes function and

$$
\kappa[\nu](\lambda)=\exp \left\{-\int_{-q}^{q} \frac{v(\lambda)-v(\mu)}{\lambda-\mu} \mathrm{d} \mu\right\}
$$

Finally, we agree upon,

$$
\begin{aligned}
& \mathcal{R}_{N, n}\left(\begin{array}{c}
\left\{\mu_{p_{a}}\right\} ;\left\{p_{a}\right\} \\
\left\{\mu_{h_{a}}\right\} ;\left\{h_{a}\right\}
\end{array}\right)[F]=\prod_{a=1}^{n}\left\{\frac{\left.\varphi\left(\mu_{h_{a}}, \mu_{h_{a}}\right) \varphi\left(\mu_{p_{a}}, \mu_{p_{a}}\right) \mathrm{e}^{\aleph\left(\mu_{p_{a}}\right.}\right)}{\left.\varphi\left(\mu_{p_{a}}, \mu_{h_{a}}\right) \varphi\left(\mu_{h_{a}}, \mu_{p_{a}}\right) \mathrm{e}^{\aleph\left(\mu_{h_{a}}\right)}\right\}} \operatorname{det}_{n}^{2}\left[\frac{1}{h_{a}-p_{b}}\right] \prod_{a=1}^{n}\left(\frac{\sin \left[\pi v\left(\mu_{h_{a}}\right)\right]}{\pi}\right)^{2}\right. \\
& \times \frac{\prod_{a<b}^{n} \varphi^{2}\left(\mu_{p_{a}}, \mu_{p_{b}}\right) \varphi^{2}\left(\mu_{h_{a}}, \mu_{h_{b}}\right)}{\prod_{a \neq b}^{n} \varphi^{2}\left(\mu_{p_{a}}, \mu_{h_{b}}\right)} \\
& \cdot \Gamma^{2}\left(\begin{array}{c}
\left.\left\{p_{a}-N-1+v\left(\mu_{p_{a}}\right)\right\},\left\{p_{a}\right\},\left\{N+2-h_{a}-v\left(\mu_{h_{a}}\right)\right\},\left\{h_{a}+v\left(\mu_{h_{a}}\right)\right\}\right) . \\
\left\{p_{a}-N-1\right\},\left\{p_{a}+v\left(\mu_{p_{a}}\right)\right\},\left\{N+2-h_{a}\right\},\left\{h_{a}\right\}
\end{array}\right.
\end{aligned}
$$

There

$$
\aleph(\omega)=2 v(\omega) \ln \left(\frac{\varphi(\omega, q)}{\varphi(\omega,-q)}\right)+2 \int_{-q}^{q} \frac{v(\lambda)-v(\omega)}{\lambda-\omega} \mathrm{d} \lambda \quad \text { and } \quad \varphi(\lambda, \mu)=2 \pi \frac{\lambda-\mu}{p(\lambda)-p(\mu)} .
$$


Above, we have used the standard hypergeometric-type representation for products of $\Gamma$-functions

$$
\Gamma\left(\begin{array}{l}
\left\{a_{k}\right\} \\
\left\{b_{k}\right\}
\end{array}\right)=\prod_{k=1}^{n} \frac{\Gamma\left(a_{k}\right)}{\Gamma\left(b_{k}\right)} .
$$

\section{The Fredholm determinants}

In this section, we provide a way to define Fredholm determinants entering in the leading asymptotic behavior of the properly normalized form factors of the conjugated field in the case where the contour $\mathscr{C}_{q}$, as it has been described previously, does not exist. Actually, this definition holds as well in the case of complex valued rapidities. Prior to stating the result, we need to introduce some notations. Given $\delta>0$ and $\epsilon>0$, we introduce

$$
U_{\delta}=\{z \in \mathbb{C}:|\Im(z)|<\delta\} \quad \text { and } \quad \mathscr{K}_{\epsilon}=\{z \in \mathbb{C}:|\Im(z)|<\delta / 2 \text { and }|\Re(z)|<q+\epsilon\} .
$$

Finally, given $\beta_{0} \in \mathbb{C}$, we denote

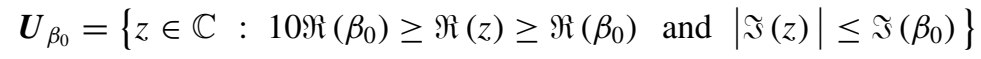

and agree that $D_{0, \epsilon}$ stands for the open disk of radius $\epsilon$ that is centered at 0 . Also, $\bar{S}$ refers to the closure of the set $S$.

Proposition 4.1: Let $m \in \mathbb{N}$ be fixed and $\epsilon, \delta>0$ be small enough. Assume that one is given two holomorphic function $v$ and $h$ on $U_{2 \delta}$, such that

$$
h\left(U_{2 \delta}\right) \subset\{z: \Re(z)>0\} \text { and } z \mapsto \Im(h(z)) \text { is bounded on } U_{2 \delta} .
$$

Then, there exists

- $\beta_{0} \in \mathbb{C}$ with $\Re\left(\beta_{0}\right)>0$ large enough and $\Im\left(\beta_{0}\right)>0$ small enough;

- $\gamma_{0}>0$ but small enough;

- a small counterclockwise loop $\mathscr{C}_{q}$ around $\overline{\mathscr{K}}_{\epsilon}$ and in $U_{2 \delta}$;

such that given $v_{\beta}(\lambda)=v(\lambda)+i \beta h(\lambda)$, one has

$$
\mathrm{e}^{-2 i \pi \gamma(\nu+i \beta h)(\lambda)}-1 \neq 0 \quad \forall \lambda \quad \text { on and inside } \mathscr{C}_{q} \quad \text { and } \quad \text { uniformly in }(\beta, \gamma) \in \boldsymbol{U}_{\beta_{0}} \times D_{0, \gamma_{0}} .
$$

Moreover, given an integral kernel $U\left[\gamma v_{\beta}\right]\left(\left\{\mu_{p_{a}}\right\}_{1}^{n},\left\{\mu_{h_{a}}\right\}_{1}^{n}\right)\left(\omega, \omega^{\prime}\right)$ as defined by (4.16), the function

$$
\begin{aligned}
\mathcal{F}(z)= & G\left(1-\gamma v_{\beta}(-q)\right) G\left(2+\gamma v_{\beta}(q)\right) \prod_{a=1}^{n}\left(\mathrm{e}^{-2 i \pi \gamma v_{\beta}\left(\mu_{h_{a}}\right)}-1\right) \\
& \cdot \operatorname{det}_{\mathscr{C}_{q}}\left[I+\gamma U\left[\gamma v_{\beta}\right]\left(\left\{\mu_{p_{a}}\right\}_{1}^{n},\left\{\mu_{h_{a}}\right\}_{1}^{n}\right)\right]
\end{aligned}
$$

is holomorphic in $z=\left(\left\{\mu_{p_{a}}\right\}_{1}^{n},\left\{\mu_{h_{a}}\right\}_{1}^{n}, \beta, \gamma\right)$ belonging to $\mathcal{D}_{0}=U_{\delta}^{n} \times \mathscr{K}_{\epsilon}^{n} \times \widetilde{\boldsymbol{U}}_{\beta_{0}} \times D_{0, \gamma_{0}}$, this uniformly in $0 \leq n \leq m$.

It admits a (unique) analytic continuation to $\mathcal{D}=U_{\delta}^{n} \times \mathscr{K}_{\epsilon}^{n} \times\{z \in \mathbb{C}: \Re(z) \geq-\epsilon\}$ $\times D_{0,1+\epsilon}$.

Proof: We beginning by proving the first statement. We choose a small counterclockwise loop $\mathscr{C}_{q}$ around $\overline{\mathscr{K}}_{\epsilon}$ and in $U_{2 \delta}$. We denote by $K$ the compact, such that $\partial K=\mathscr{C}_{q}$. Then one has, $\forall \lambda \in K$,

$$
\Im\left(v_{\beta}(\lambda)\right) \geq-\sup _{K}|\Im(v(\lambda))|-\Im\left(\beta_{0}\right) \sup _{K}|\Im(h(\lambda))|+\Re\left(\beta_{0}\right) \inf _{K}[\Re(h(\lambda))] .
$$


Thus, $\Im\left(v_{\beta}(\lambda)\right)>0$ provided that $\beta \in \boldsymbol{U}_{\beta_{0}}$, with $\Im\left(\beta_{0}\right)=\delta$ and $\Re\left(\beta_{0}\right)$, such that

$$
\Re\left(\beta_{0}\right)>\frac{1}{\inf _{K}[\Re(h(\lambda))]}\left[\sup _{K}|\Im(\nu(\lambda))|+\Im\left(\beta_{0}\right) \sup _{K}|\Im(h(\lambda))|\right] .
$$

Then, $\gamma_{0}$ is chosen such that

$$
0<\gamma_{0} \leq \frac{1}{2}\left[\sup _{K}|v(\lambda)|+\left(10 \Re\left(\beta_{0}\right)+\Im\left(\beta_{0}\right)\right) \sup _{K}|h(\lambda)|\right] .
$$

It is then easy to show that, for such a $\gamma_{0}$, one has $\gamma_{0} \sup _{K}\left|v_{\beta}(\lambda)\right| \leq 1 / 2$. This estimate holds uniformly in $(\beta, \gamma) \in \boldsymbol{U}_{\beta_{0}} \times D_{0, \gamma_{0}}$. As a consequence, the function $\lambda \mapsto \varphi(\lambda, \beta, \gamma)$ with

$$
\varphi(\lambda, \beta, \gamma)=\mathrm{e}^{-2 i \pi \gamma v_{\beta}(\lambda)}-1
$$

has no zeroes in $K$.

Hence, the integral kernel of the operator $\gamma U\left[\gamma v_{\beta}\right]\left(\left\{\mu_{p_{a}}\right\}_{1}^{n},\left\{\mu_{h_{a}}\right\}_{1}^{n}\right)$ is smooth on $\mathscr{C}_{q} \times \mathscr{C}_{q}$. As $\mathscr{C}_{q}$ is compact, this aforementioned operator is trace class on $L^{1}\left(\mathscr{C}_{q}\right)$. Moreover, it depends holomorphically on $\left\{\mu_{p_{a}}\right\}_{1}^{n} \in U_{\delta}^{n},\left\{\mu_{h_{a}}\right\}_{1}^{n} \in \mathscr{K}_{\epsilon}^{n}$, and $(\beta, \gamma) \in \boldsymbol{U}_{\beta_{0}} \times D_{0, \gamma_{0}}$. Standard properties of operator determinants ${ }^{20}$ then ensure that $\mathcal{F}(\boldsymbol{z})$, as defined in (4.28) is holomorphic in $z \in \mathcal{D}_{0}$. We remind that, for the purpose of this section, a bold letter $z$ refers to vectors of the type $z$ $=\left(\left\{\mu_{p_{a}}\right\}_{1}^{n},\left\{\mu_{h_{a}}\right\}_{1}^{n}, \beta, \gamma\right)$.

Let $A$ be the set

$$
A=\left\{z \in \mathcal{D}: \prod_{\epsilon= \pm} \gamma^{-1}\left(\mathrm{e}^{-2 i \pi \gamma v_{\beta}(\epsilon q)}-1\right) \prod_{a=1}^{n} \gamma^{-1}\left(\mathrm{e}^{-2 i \pi \gamma v_{\beta}\left(\mu_{h_{a}}\right)}-1\right)=0\right\} .
$$

By definition $A$ is an analytic set. Moreover, since it is realized as the locus of zeroes of a single, non-zero, holomorphic function on $\mathcal{D}$, it has at least codimension 1 (cf. Ref. 19).

It follows from the first part of the proof that $\mathcal{F}(z)$ is indeed well defined on $\mathcal{D}_{0}$. It can be naturally extended to a holomorphic function on the set $\mathcal{D} \backslash A$ by deforming the original contour $\mathscr{C}_{q}$ in such a way that the zeroes of $\mathrm{e}^{-2 i \pi \gamma v_{\beta}(\lambda)}-1$ are not surrounded by $\mathscr{C}_{q}$, whereas the points $\pm q$ and $\mu_{h_{1}}, \ldots, \mu_{h_{n}}$ are surrounded by it. Such a deformation is always possible as, on the one hand, $z \notin A$ so that the zeroes of $\mathrm{e}^{-2 i \pi \gamma \nu_{\beta}(\lambda)}-1$ are indeed distinct from the points $\pm q$ and $\mu_{h_{1}}, \ldots, \mu_{h_{n}}$. On the other hand, it is allowed to deform the contour by applying the Cauchy theorem: the integrand is a holomorphic function on the region, where the deformation of interest takes place. Indeed, the only terms that are not explicitly holomorphic in the integral kernel (4.16) are the various Cauchy transforms. However, as $v_{\beta}$ is holomorphic on $U_{2 \delta}$, the cut of the Cauchy transform can be deformed within $U_{2 \delta}$ as long as it keeps its endpoints on $\pm q$.

It thus remains to show that $\mathcal{F}(z)$ can be analytically continued through $A$. For this, it is enough to show that given any $z^{(0)} \in A$, there exists an open neighborhood $U$ of $z^{(0)}$, such that setting $W=(\mathcal{D} \backslash A) \cap U, \mathcal{F}_{\mid W}$ is bounded.

We parameterize $\boldsymbol{z}^{(0)} \in A$ as $\boldsymbol{z}^{(0)}=\left(\left\{\mu_{p_{a}}^{(0)}\right\}_{1}^{n},\left\{\mu_{h_{a}}^{(0)}\right\}_{1}^{n}, \beta^{(0)}, \gamma^{(0)}\right)$. This means that if $\rho \in \mathcal{D} \backslash A$ and is sufficiently close to $z^{(0)}$, there exists zeroes (not necessarily distinct) $z_{1}(\rho), \ldots, z_{\ell}(\rho)$ of $\lambda \mapsto \varphi(\lambda, \beta, \gamma)$ that will approach $\pm q$ or $\mu_{h_{1}}^{(0)}, \ldots, \mu_{h_{n}}^{(0)}$ in the limit $\rho \rightarrow z^{(0)}$ in $\mathcal{D} \backslash A$

Indeed, the zeroes of a holomorphic function form discrete sets. Hence, there exists a contour $\Gamma_{z^{(0)}}$ consisting of small counterclockiwse circles around $\pm q$ and $\mu_{h_{1}}^{(0)}, \ldots, \mu_{h_{n}}^{(0)}$ such that $\varphi\left(\lambda, \beta^{(0)}, \gamma^{(0)}\right) \neq 0$ for all $\lambda \in \Gamma_{z^{(0)}}$.

The function $(\lambda, \beta, \gamma) \mapsto \varphi(\lambda, \beta, \gamma)$ is continuous and $\Gamma_{z^{(0)}}$ is compact. Hence, there exists an open neighborhood $B^{(0)}$ of $\left(\beta^{(0)}, \gamma^{(0)}\right)$ in $\mathbb{C}^{2}$, such that $\varphi(\lambda, \beta, \gamma) \neq 0$ for any $\lambda \in \Gamma_{z^{(0)}}$ and $(\beta, \gamma) \in B^{(0)}$. As a consequence, we get that for any $(\beta, \gamma) \in B^{(0)}$, the number of zeroes (counted with their multiplicities) of $\lambda \mapsto \varphi(\lambda, \beta, \gamma)$ is constant and equal to some integer $\ell$.

Let $V_{0}$ be an open set contained in the bounded connected component of $\mathbb{C} \backslash \Gamma_{z^{(0)}}$ and let $\rho=\left(\left\{\mu_{p_{a}}\right\}_{1}^{n},\left\{\mu_{h_{a}}\right\}_{1}^{n}, \beta, \gamma\right) \in \mathcal{D} \backslash A$ be such that $\mu_{h_{a}} \in V_{0}$ for any $a=1, \ldots, n$ and $(\beta, \gamma) \in B^{(0)}$. As $\rho \in \mathcal{D} \backslash A$, we necessarily have that the zeroes of $\lambda \mapsto \varphi(\lambda, \beta, \gamma)$ all differ from $\pm q$ and $\mu_{h_{a}}$, $a=1, \ldots, n$. By deforming, if necessary, the initially introduced contour $\mathscr{C}_{q}$, we can represent the 
Fredholm determinant by its Fredholm series

$$
\operatorname{det}_{\mathscr{C}_{q}}\left[I+\gamma U\left[\gamma v_{\beta}\right]\left(\left\{\mu_{p_{a}}\right\}_{1}^{n} ;\left\{\mu_{h_{a}}\right\}_{1}^{n}\right)\right]=\sum_{m \geq 0} \frac{1}{m !} \int_{\mathscr{C}_{q}} \mathrm{~d}^{n} \omega \operatorname{det}_{m}\left[U_{\beta}\left(\omega_{a}, \omega_{b}\right)\right] \prod_{a=1}^{m} \frac{\gamma}{\mathrm{e}^{-2 i \pi \gamma v_{\beta}\left(\omega_{a}\right)}-1}
$$

where we have set

$$
\begin{aligned}
U_{\beta}\left(\omega, \omega^{\prime}\right)= & \frac{-1}{2 \pi} \frac{\omega-q}{\omega-q+i c} \prod_{a=1}^{n}\left\{\frac{\left(\omega-\mu_{p_{a}}\right)\left(\omega-\mu_{h_{a}}+i c\right)}{\left(\omega-\mu_{h_{a}}\right)\left(\omega-\mu_{p_{a}}+i c\right)}\right\} \\
& \cdot \mathrm{e}^{C\left[2 i \pi \gamma v_{\beta}\right](\omega)-C\left[2 i \pi \gamma v_{\beta}\right](\omega+i c)} K\left(\omega-\omega^{\prime}\right) .
\end{aligned}
$$

We set $\tilde{\mathscr{C}}=\mathscr{C}_{q} \cup \Gamma_{z^{(0)}}$. Due to the symmetry of the integrand, we may carry out the substitution

$$
\frac{1}{m !} \int_{\widetilde{\mathscr{C}} \cup\left\{-\Gamma_{\left.z^{(0)}\right\}}\right.} \mathrm{d}^{m} \omega=\sum_{s=0}^{m} \frac{1}{s !(m-s) !} \int_{\widetilde{\mathscr{C}}} \mathrm{d}^{m-s} \omega \int_{\left\{-\Gamma_{\left.z^{(0)}\right\}}\right.} \prod_{j=1}^{s} \mathrm{~d} \omega_{m-j+1} .
$$

Note that $-\Gamma_{z^{(0)}}$ appearing above stands for the contour $\Gamma_{z^{(0)}}$ but endowed with the opposite orientation. Further, notice that for any symmetric function $f\left(\omega_{1}, \ldots, \omega_{s}\right)$ that is holomorphic in a neighborhood of the points $z_{j}(\rho)$ and vanishing on the diagonals $\left(\omega_{\ell}=\omega_{p}\right.$ for $\left.\ell \neq p\right)$, one has

$$
\begin{aligned}
\int_{-\Gamma_{z^{(0)}}} f\left(\omega_{1}, \ldots, \omega_{s}\right) \prod_{a=1}^{s} \frac{\gamma}{\mathrm{e}^{-2 i \pi \gamma v_{\beta}\left(\omega_{s}\right)}-1} \cdot \mathrm{d}^{s} \omega=s ! & \sum_{\substack{\llbracket 1 ; \ell \prod=\alpha_{-} \cup \alpha_{+} \\
\left|\alpha_{+}\right|=s}} f\left(z_{\alpha_{1}}(\boldsymbol{\rho}), \ldots, z_{\alpha_{s}}(\boldsymbol{\rho})\right) \\
& \times \prod_{j=1}^{s} \frac{1}{v_{\beta}^{\prime}\left(z_{\alpha_{j}}(\boldsymbol{\rho})\right)}
\end{aligned}
$$

Above, the sum runs through all the partitions of $\llbracket 1 ; \ell \rrbracket$ into two disjoint subsets $\alpha_{+} \cup \alpha_{-}$, such that $\alpha_{+}=\left(\alpha_{1}, \ldots, \alpha_{s}\right)$ contains $s$ elements, i.e., $\left|\alpha_{+}\right|=s$. Note that we have here tacitly assumed that all of the roots are simple. The case of multiple roots can then be obtained by carrying out a limiting procedure on (4.37).

Therefore, we obtain the below representation for the Fredholm determinant

$$
\begin{gathered}
\operatorname{det}_{\mathscr{C}_{q}}\left[I+\gamma U\left[\gamma v_{\beta}\right]\right]=\sum_{m \geq 0} \sum_{s=0}^{\min (m, p)} \frac{(-1)^{m}}{(m-s) !} \sum_{\substack{\llbracket 1 ; p \rrbracket=\alpha_{-} \cup \alpha_{+} \\
\left|\alpha_{+}\right|=s}} \int_{\widetilde{\mathscr{C}}} \frac{\mathrm{d}^{m-s} \omega}{(2 \pi)^{m}} \operatorname{det}_{m}\left[K\left(\omega_{k}-\omega_{j}\right)\right] \\
\prod_{b=1}^{s}\left\{\frac{z_{\alpha_{b}}(\boldsymbol{\rho})-q}{z_{\alpha_{b}}(\boldsymbol{\rho})-q+i c} \prod_{a=1}^{n}\left[\frac{\left(z_{\alpha_{b}}(\boldsymbol{\rho})-\mu_{p_{a}}\right)\left(z_{\alpha_{b}}(\boldsymbol{\rho})-\mu_{h_{a}}+i c\right)}{\left(z_{\alpha_{b}}(\boldsymbol{\rho})-\mu_{h_{a}}\right)\left(z_{\alpha_{b}}(\boldsymbol{\rho})-\mu_{p_{a}}+i c\right)}\right] \frac{\mathrm{e}^{C\left[2 i \pi \gamma v_{\beta}\right]\left(z_{\alpha_{b}}(\boldsymbol{\rho})\right)-C\left[2 i \pi \gamma v_{\beta}\right]\left(z_{\alpha_{b}}(\boldsymbol{\rho})+i c\right)}}{v_{\beta}^{\prime}\left(z_{\alpha_{b}}(\boldsymbol{\rho})\right)}\right\} \\
\left.\prod_{k=1}^{m-s}\left\{\frac{\omega_{k}-q}{\omega_{k}-q+i c} \prod_{a=1}^{n}\left[\frac{\left(\omega_{k}-\mu_{p_{a}}\right)\left(\omega_{k}-\mu_{h_{a}}+i c\right)}{\left(\omega_{k}-\mu_{h_{a}}\right)\left(\omega_{k}-\mu_{p_{a}}+i c\right.}\right)\right] \gamma \frac{\mathrm{e}^{C\left[2 i \pi \gamma v_{\beta}\right]\left(\omega_{k}\right)-C\left[2 i \pi \gamma v_{\beta}\right]\left(\omega_{k}+i c\right)}}{\mathrm{e}^{-2 i \pi v_{\beta}\left(\omega_{k}\right)}-1}\right\}
\end{gathered}
$$

and we agree upon the shorthand notation $\omega_{m-j+1}=z_{\alpha_{j}}(\boldsymbol{\rho})$ for $j=1, \ldots, s$ for the determinant that occurs in the first line.

For any fixed $\rho$, one has the decomposition in respect to zeroes,

$$
\mathrm{e}^{-2 i \pi \gamma v_{\beta}(\omega)}-1=\prod_{a=1}^{\ell}\left(\omega-z_{a}(\rho)\right) \cdot V_{\beta, \gamma}(\omega)
$$


with $V_{\beta, \gamma}(\omega)$ a holomorphic function on $\mathscr{K}_{\epsilon}$ that has no zeroes on $\tilde{\mathscr{C}}$ and $V_{0}$ uniformly in $(\beta, \gamma)$ $\in B^{(0)}$. It thus follows that the function

$$
\prod_{a=1}^{n}\left\{\left(\mathrm{e}^{-2 i \pi \gamma v_{\beta}\left(\mu_{h_{a}}\right)}-1\right) \prod_{b=1}^{s} \frac{1}{z_{\alpha_{b}}(\rho)-\mu_{h_{a}}}\right\}
$$

is bounded on $\rho$ belonging to $(\mathcal{D} \backslash A) \cap \mathscr{U}$ with $\mathscr{U}=U_{\delta}^{n} \times V_{0}^{n} \times B^{(0)}$. Note that the above reasoning holds for simple roots. In the case of multiple roots, one should first carry out a limiting procedure on the level of (4.38), which will lead to the appearance of derivatives. The final conclusion, however, still holds. We leave these details to the reader.

It only remains to focus on the Cauchy transforms. The latter can be represented as

$$
\mathrm{e}^{C\left[2 i \pi \gamma \nu_{\beta}\right](\omega)}=\exp \left\{\int_{-q}^{q} \gamma \frac{\nu_{\beta}(\lambda)-v_{\beta}(\omega)}{\lambda-\omega} \mathrm{d} \lambda\right\}\left(\frac{\omega-q}{\omega+q}\right)^{\gamma v_{\beta}(\omega)} .
$$

As a consequence, the only divergencies that can arise from the Cauchy transform are located at $\omega= \pm q$.

If there exists a $k$ such that $z_{k}\left(z^{(0)}\right)= \pm q$, then there exists $\ell_{k} \in \mathbb{Z}$ such that $\gamma v_{\beta}\left(z_{k}\left(z^{(0)}\right)\right)=\ell_{k}$. As a consequence, the Cauchy transforms occurring in the second line of (4.38) may introduce divergent contributions. Yet, since the Barnes function has a simple zero of order $p+1$ at $-p$, with $p \in \mathbb{N}$, it is easy to see that

$$
G\left(1-\gamma v_{\beta}(-q)\right) G\left(2+\gamma v_{\beta}(q)\right)\left(z_{k}(\boldsymbol{\rho})-q\right) \cdot \prod_{k=1}^{\ell}\left(\frac{z_{k}(\boldsymbol{\rho})-q}{z_{k}(\boldsymbol{\rho})+q}\right)^{\gamma v_{\beta}\left(z_{k}(\boldsymbol{\rho})\right)}
$$

is bounded for $\rho \in(\mathcal{D} \backslash A) \cap \mathscr{U}$. The fact that all other terms in (4.38) are bounded is evident. The theorem then follows after applying the analytic continuation theorem in many variables. ${ }^{19}$

\section{CONCLUSION}

In this paper, we proved the convergence towards naturally associated quantities in the continuous model of scalar products and form factors arising in the lattice discretizations of the NLSM. This provides the last missing step towards the proof of determinant-based representations for these object in the continuum. Our approach was based on a generalization and simplification of the techniques proposed in Ref. 5. We have also provided a unambiguous procedure for defining the class of Fredholm determinants that occurs in the large volume limit of properly normalized form factors in integrable models, this on the example of the NLSM. It would be quite natural to continue this kind of considerations for lattice discretizations of more involved models such as the Sine-Gordon model. However, in this case additional complications will arise due to the non-conservations of the number of particles.

\section{ACKNOWLEDGMENTS}

I acknowledge the support of the EU Marie-Curie Excellence Grant No. MEXT-CT-2006042695. I would like to thank N. Kitanine, J.-M. Maillet, N. Slavnov, J. Teschner, and V. Terras for stimulating discussions. 


\section{APPENDIX: PROOF OF THEOREM}

\section{Combinatorial representation of the eigenstates}

Lemma 1.1: Let $\left\{\lambda_{j}\right\}$ be $N$ generic parameters, then the below representation holds

$$
B\left(\lambda_{1}\right) \ldots B\left(\lambda_{N}\right)|0\rangle=\sum_{\substack{1 \leq n_{1} \leq \\ \cdots \leq n_{N} \leq M}} \prod_{a=1}^{M} \frac{1}{\left(\# \Gamma_{a}\right) !} \cdot f_{\{\lambda\}}\left(n_{1}, \ldots, n_{N}\right) \cdot \beta_{n_{1}}^{*} \ldots \beta_{n_{N}}^{*}|0\rangle,
$$

where we agree upon $\beta_{k}^{*}=-i \sqrt{c} \chi_{k}^{*} \rho_{Z_{k}}$ and $\Gamma_{k}=\left\{\ell: n_{\ell}=k\right\}, k=1, \ldots, M$. In (Al), we have set

$$
\begin{array}{r}
f_{\{\lambda\}}\left(n_{1}, \ldots, n_{N}\right)=\sum_{\sigma \in \mathfrak{S}_{N} a<b} \prod_{\frac{\lambda_{\sigma(a)}}{a}-\lambda_{\sigma(b)}+i c \operatorname{sgn}\left(n_{b}-n_{a}\right)}^{N} \prod_{a=1}^{N}\left\{\alpha\left(\lambda_{\sigma(a)}\right)\right\}^{\left[\frac{n_{a}-1}{2}\right]}\left\{\bar{\alpha}\left(\lambda_{\sigma(a)}\right)\right\}^{\left[\frac{M-n_{a}}{2}\right]} \\
\times \prod_{a=1}^{N}\left\{1-(-1)^{n_{a}} \Delta\left(\frac{c}{4}-i \frac{\lambda_{\sigma(a)}}{2}\right)\right\} . \quad \text { (A2) }
\end{array}
$$

The sign function appearing above has been defined in (2.25), [.] stands for the floor function and we agree upon

$$
\alpha(\lambda)=\left(1-\frac{c \Delta}{4}+i \frac{\lambda \Delta}{4}\right)\left(1+\frac{c \Delta}{4}+i \frac{\lambda \Delta}{4}\right) \quad \text { and } \quad \bar{\alpha}(\lambda)=\alpha(-\lambda) .
$$

Proof: It is a standard fact ${ }^{11}$ that, for any generic set of parameters $\left\{\lambda_{j}\right\}$, the action of a product of $B$ operators on the pseudo-vacuum can be expressed as a sum over all the possible partitions of

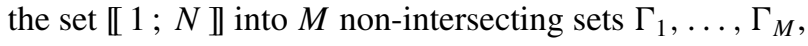

$$
\begin{aligned}
\prod_{k=1}^{N} B\left(\lambda_{k}\right)|0\rangle= & \sum_{\substack{\llbracket 1 ; N \rrbracket \\
=\cup_{k=1}^{M} \Gamma_{k}}} \prod_{\ell=2}^{M} \prod_{m=1}^{\ell-1}\left\{\prod_{a \in \Gamma_{m}} \prod_{b \in \Gamma_{\ell}} \frac{\lambda_{a}-\lambda_{b}+i c}{\lambda_{a}-\lambda_{b}} \prod_{a \in \Gamma_{\ell}}\left(Z_{m}+i \frac{\Delta \lambda_{a}}{2}\right)\right. \\
& \left.\times \prod_{b \in \Gamma_{m}}\left(Z_{\ell}-i \frac{\Delta \lambda_{b}}{2}\right)\right\} \cdot \prod_{a=1}^{M}\left(\beta_{a}^{*}\right)^{\# \Gamma_{a}}|0\rangle .
\end{aligned}
$$

We stress that in the above decomposition, the ordering of the partition counts, i.e., $\{1,2,3\} \cup\{\emptyset\}$ is different from $\{\varnothing\} \cup\{1,2,3\}$. Also, we have denoted by $\# \Gamma_{a}$ the cardinality of the set $\Gamma_{a}$.

Note that there is a one-to-one correspondence between the set of all such partitions and choices of $N$ integers $n_{1}, \ldots, n_{N}$ in $\llbracket 1 ; M \rrbracket$ by the formula $\Gamma_{k}=\left\{\ell: n_{\ell}=k\right\}$. One can thus recast the sums in (A4) as ones over such choices of integers. Namely,

$$
\prod_{k=1}^{N} B\left(\lambda_{k}\right)|0\rangle=\sum_{\substack{1 \leq n_{1} \leq \\ \cdots \leq n_{N} \leq M}} \prod_{a=1}^{M} \frac{1}{\left(\# \Gamma_{a}\right) !} \cdot f_{\{\lambda\}}\left(n_{1}, \ldots n_{N}\right) \cdot \beta_{n_{1}}^{*} \ldots \beta_{n_{M}}^{*}|0\rangle,
$$

where

$$
\begin{gathered}
f_{\{\lambda\}}\left(n_{1}, \ldots n_{N}\right)=\sum_{\sigma \in \mathfrak{S}_{N}} \prod_{\ell=2}^{M} \prod_{m=1}^{\ell-1}\left\{\prod_{\substack{a: \\
n_{\sigma^{-1}(a)}=m}} \prod_{\substack{b: \\
n_{\sigma-1}(b)}}\left\{\frac{\lambda_{a}-\lambda_{b}+i c}{\lambda_{a}-\lambda_{b}}\right\}\right. \\
\left.\prod_{\substack{a: \\
n_{\sigma-1}(a)}}\left(Z_{m}+i \frac{\Delta \lambda_{a}}{2}\right) \prod_{\substack{b: \\
n_{\sigma-1}(b)}}\left(Z_{\ell}-i \frac{\Delta \lambda_{b}}{2}\right)\right\} .
\end{gathered}
$$


In (A5), we have recast the sum over $n_{a} \in \llbracket 1 ; M \rrbracket$ into one over the ordered choices of integers $1 \leq n_{1} \leq \cdots \leq n_{N} \leq M$, this by introducing an additional sum over permutations $\sigma \in \mathfrak{S}_{N}$ in (A6). However, so as not to count elements twice, for each given choice of integers $1 \leq n_{1} \leq \cdots \leq n_{N}$ $\leq M$, we divide by $\prod_{a=1}^{N}\left(\# \Gamma_{a}\right)$ !. Indeed, the permutation group leaves the diagonals unaltered, and each of such diagonals corresponds to elements in the set $\Gamma_{a}$, occurring in the partition $\cup \Gamma_{a}$.

Then, it is enough to observe that

$$
\prod_{\ell=2}^{M} \prod_{m=1}^{\ell-1} \prod_{\substack{a: \\ n_{\sigma^{-1}(a)}=m}} \prod_{\substack{b: \\ n_{\sigma^{-1}(b)}=\ell}}\left\{\frac{\lambda_{a}-\lambda_{b}+i c}{\lambda_{a}-\lambda_{b}}\right\}=\prod_{a<b}^{N} \frac{\lambda_{\sigma(a)}-\lambda_{\sigma(b)}+i c \operatorname{sgn}\left(n_{b}-n_{a}\right)}{\lambda_{\sigma(a)}-\lambda_{\sigma(b)}}
$$

and

$$
\begin{aligned}
& \prod_{\ell=2}^{M} \prod_{m=1}^{\ell-1} \prod_{\substack{a: \\
n_{\sigma^{-1}(a)}=\ell}}\left(Z_{m}+i \frac{\Delta \lambda_{a}}{2}\right) \prod_{\substack{b: \\
n_{\sigma^{-1}(b)}=m}}\left(Z_{\ell}-i \frac{\Delta \lambda_{b}}{2}\right) \\
& \quad=\prod_{a=1}^{N}\left\{\alpha\left(\lambda_{\sigma(a)}\right)\right\}^{\left[\frac{n_{a}-1}{2}\right]}\left\{\bar{\alpha}\left(\lambda_{\sigma(a)}\right)\right\}^{\left[\frac{M-n_{a}}{2}\right]} \times \prod_{a=1}^{N}\left\{1-(-1)^{n_{a}} \Delta\left(\frac{c}{4}-i \frac{\lambda_{\sigma(a)}}{2}\right)\right\} .
\end{aligned}
$$

In fact, given a solution $\left\{\lambda_{\ell_{a}}\right\}_{1}^{N}$ of the Bethe equations (2.21), the associated function $f_{\{\lambda\}}$ as defined in (A2) is bounded uniformly in $\Delta$ small enough. This is an important property in respect to taking the $\Delta \rightarrow 0$ limit.

Lemma 1.2: Let $\left\{\lambda_{\ell_{a}}\right\}_{1}^{N}$ be a solution of the Bethe equations (2.21) associated with the choice of integers $\ell_{1}<\cdots<\ell_{N}$. Then, there exists $\Delta_{0}$ small enough and a constant $C_{\left\{\ell_{a}\right\}}$ solely depending on $N, L, \Delta_{0}$, and the choice of integers $\left\{\ell_{a}\right\}$, such that

$$
\left|f_{\{\lambda\}}\left(n_{1}, \ldots, n_{N}\right)\right| \leq C_{\left\{\ell_{a}\right\}} \quad \text { uniformly } \Delta \in\left[0 ; \Delta_{0}\right]
$$

where $f_{\{\lambda\}}$ has been defined in (A2).

Proof: It follows from the continuity in $\Delta$ on $\left[0 ; \Delta_{0}\right]$ of $\Delta \mapsto \lambda_{\ell_{a}}, a=1, \ldots, N$, (cf. Sec. I C ) that the function $\Delta \mapsto \min _{a<b}\left|\lambda_{\ell_{a}}-\lambda_{\ell_{b}}\right|$ is continuous on $\left[0 ; \Delta_{0}\right]$. Thus, it attains its minimum at some $\widetilde{\Delta} \in\left[0 ; \Delta_{0}\right]$. However, in virtue of the repulsion principle (2.20), this minimum must be strictly positive, and thus

$$
m_{\left\{\ell_{a}\right\}}=\inf _{\Delta \in\left[0 ; \Delta_{0}\right]} \min _{a<b}\left|\lambda_{\ell_{a}}-\lambda_{\ell_{b}}\right|>0 .
$$

For each $\Delta$, the associated parameters $\lambda_{\ell_{a}}$ are bounded. Hence, the function $\Delta \mapsto \max _{a}\left|\lambda_{\ell_{a}}\right|$ is well defined and continuous in $\Delta \in\left[0 ; \Delta_{0}\right]$. As argued before, this implies that

$$
M_{\left\{\ell_{a}\right\}}=\sup _{\Delta \in\left[0 ; \Delta_{0}\right]} \max _{a}\left|\lambda_{\ell_{a}}\right|<+\infty .
$$

Hence, given any choice of integers $1 \leq n_{1} \leq \cdots \leq n_{N} \leq M$,

$$
\left|\prod_{n_{a}}^{N}\left\{1-(-1)^{n_{a}} \Delta\left(\frac{c}{4}-i \frac{\lambda_{\sigma(a)}}{2}\right)\right\}\right| \leq\left(1+\frac{\Delta}{4}\left(c+2 M_{\left\{\ell_{a}\right\}}\right)\right)^{N}
$$

and, for any $a \in \llbracket 1 ; n \rrbracket$,

$$
\left|\alpha\left( \pm \lambda_{a}\right)\right| \leq \mathrm{e}^{M_{\left\{\ell_{a}\right\}} \Delta+\frac{c \Delta}{2}}
$$

Thus, as $M \Delta=L$,

$$
\left|\prod_{a=1}^{N}\left\{\alpha\left(\lambda_{\sigma(a)}\right)\right\}^{\left[\frac{n_{a}-1}{2}\right]}\left\{\bar{\alpha}\left(\lambda_{\sigma(a)}\right)\right\}^{\left[\frac{M-n_{a}}{2}\right]}\right| \leq \mathrm{e}^{\left(M_{\left\{\ell_{a}\right\}}+\frac{c}{2}\right) \Delta \sum_{a=1}^{N}\left[\frac{n_{a}-1}{2}\right]+\left[\frac{M-n_{a}}{2}\right]} \leq \mathrm{e}^{\left(M_{\left\{\ell_{a}\right\}}+\frac{c}{2}\right) \frac{N L}{2}} .
$$


Last but not least

$$
\left|\prod_{a<b}^{N} \frac{\lambda_{\sigma(a)}-\lambda_{\sigma(b)}+i c \operatorname{sgn}\left(n_{b}-n_{a}\right)}{\lambda_{\sigma(a)}-\lambda_{\sigma(b)}}\right| \leq\left(\frac{2 M_{\left\{\ell_{a}\right\}}+c}{m_{\left\{\ell_{a}\right\}}}\right)^{\frac{N(N-1)}{2}} .
$$

Putting all these estimates together leads to

$$
\left|f_{\{\lambda\}}\left(n_{1}, \ldots, n_{N}\right)\right| \leq N !\left(1+\frac{\Delta_{0}}{4}\left(c+2 M_{\left\{\ell_{a}\right\}}\right)\right)^{N} \mathrm{e}^{\left(M_{\left\{\ell_{a}\right\}}+\frac{c}{2}\right) \frac{N L}{2}}\left(\frac{2 M_{\left\{\ell_{a}\right\}}+c}{m_{\left\{\ell_{a}\right\}}}\right)^{\frac{N(N-1)}{2}}
$$

uniformly in $\Delta \in\left[0 ; \Delta_{0}\right]$ and $1 \leq n_{1} \leq \cdots \leq n_{N} \leq M$.

\section{The scalar product formula} satisfy

By setting $\beta_{k}^{*}=-i \sqrt{c} \chi_{k}^{*} \rho_{Z_{k}}, \quad \beta_{k}=i \sqrt{c} \rho_{Z_{k}} \chi_{k}$, and $h_{k}=Z_{k}+\chi_{k}^{*} \chi_{k}$ it is easy to see that these

$$
\left[\beta_{k}, \beta_{n}^{*}\right]=\Delta c h_{k} \delta_{k n} \quad \text { and } \quad\left[h_{k}, \beta_{n}^{*}\right]=\frac{\Delta c}{2} \beta_{k}^{*} \delta_{k n}
$$

These commutation relations readily lead to

$$
\left\langle 0\left|\left(\beta_{k}\right)^{n} \cdot\left(\beta_{p}^{*}\right)^{m}\right| 0\right\rangle=\delta_{n, m}\left(\delta_{k, p}+\delta_{n, 0} \delta_{m, 0}\right)(\Delta c)^{n} n ! \prod_{\ell=1}^{n}\left(Z_{k}+(\ell-1) \Delta c / 4\right) .
$$

Thus, given a solution of the Bethe equations $\left\{\lambda_{\ell_{a}}\right\}_{1}^{N}$ defined by the integers $\ell_{1}<\cdots<\ell_{N}$, and a set of generic parameters $\left\{\mu_{a}\right\}_{1}^{N}$ that are bounded, and satisfy the condition $\min _{a<b}\left|\mu_{a}-\mu_{b}\right|>0$, building on the representation for the Bethe vectors (A1), one gets

$$
\begin{aligned}
\left\langle\psi\left(\left\{\mu_{a}\right\}_{1}^{N}\right) \mid \psi\left(\left\{\lambda_{\ell_{a}}\right\}_{1}^{N}\right)\right\rangle= & (\Delta c)^{N} \sum_{\substack{1 \leq n_{1} \leq \\
\cdots \leq n_{N} \leq M}} \overline{f_{\{\mu\}}}\left(n_{1}, \ldots, n_{N}\right) f_{\{\lambda\}}\left(n_{1}, \ldots, n_{N}\right) \\
& \times \prod_{a=1}^{M} \prod_{p=1}^{\# \Gamma_{a}-1} \frac{Z_{n_{a}}+p \Delta c / 4}{p+1} .
\end{aligned}
$$

Note that, to obtain (A18), we have used that

$$
\left\langle 0\left|\beta_{n_{1}^{\prime}} \ldots \beta_{n_{N}^{\prime}} \beta_{n_{1}}^{*} \ldots \beta_{n_{N}}^{*}\right| 0\right\rangle \neq 0 \quad \text { with } \quad 1 \leq n_{1}^{\prime} \leq \ldots n_{N}^{\prime} \leq M \quad \text { and } \quad 1 \leq n_{1} \leq \ldots n_{N} \leq M
$$

only if $n_{a}=n_{a}^{\prime}$ for any $a$. It is convenient to split in (A18) the contributions form the diagonals ( $n_{a}=n_{a+1}$ for some $a$ ) from those lying purely off the diagonal

$$
\left\langle\psi\left(\left\{\mu_{a}\right\}_{1}^{N}\right) \mid \psi\left(\left\{\lambda_{\ell_{a}}\right\}_{1}^{N}\right)\right\rangle=L_{1}+L_{2},
$$

where

$$
L_{1}=(\Delta c)^{N} \sum_{\substack{1<n_{1}<\\ \cdots<n_{N} \leq M}} \overline{f_{\{\mu\}}}\left(n_{1}, \ldots, n_{N}\right) f_{\{\lambda\}}\left(n_{1}, \ldots, n_{N}\right) \prod_{a=1}^{N} Z_{n_{a}}
$$

and

$$
L_{2}=(\Delta c)^{N} \sum_{k=1}^{N-1} \sum_{\substack{1 \leq n_{1} \leq \ldots \\ \leq n_{k}=n_{k+1} \leq \\ \cdots \leq n_{N} \leq M}} \overline{f_{\{\mu\}}}\left(n_{1}, \ldots, n_{N}\right) f_{\{\lambda\}}\left(n_{1}, \ldots, n_{N}\right) \prod_{a=1}^{N} \prod_{p=1}^{\# \Gamma_{a}-1} \frac{Z_{n_{a}}+p \Delta c / 4}{p+1} .
$$


The multiplicative factor in $L_{2}$ is bounded due to

$$
\left|\prod_{a=1}^{N} \prod_{p=1}^{\# \Gamma_{a}-1} \frac{Z_{n_{a}}+p \Delta c / 4}{p+1}\right| \leq \prod_{a=1}^{N} \prod_{p=1}^{\# \Gamma_{a}} \frac{1+\Delta c p / 4}{p} \leq \mathrm{e}^{N \Delta M \frac{c}{4}} .
$$

By applying Lemma 1.2 to the function $f_{\{\lambda\}}\left(n_{1}, \ldots, n_{N}\right)$ and carrying out a similar reasoning to the one of the lemma, we get $\overline{f_{\{\mu\}}}\left(n_{1}, \ldots, n_{N}\right) \leq C_{N}(\{\mu\})$, uniformly in $\Delta \in\left[0 ; \Delta_{0}\right]$ and for some $\mu_{a}$ dependent constant $C_{N}(\{\mu\})$. Hence, as $L=\Delta M$,

$$
\left|L_{2}\right| \leq N(\Delta c)^{N} M^{N-1} C_{\left\{\ell_{a}\right\}} \cdot C_{N}(\{\mu\}) \mathrm{e}^{\frac{N L}{4} c}=\mathrm{O}(\Delta) .
$$

As a consequence, $L_{2}$ does not contribute to the $\Delta \rightarrow 0$ limit of the scalar product.

It remains to treat $L_{1}$. Using that the parameters $\lambda_{\ell_{a}}$ are all continuously differentiable in respect to $\Delta$, it is readily seen that, uniformly in the choices $1<n_{1}<\cdots<n_{N} \leq M$ and $\left.\left.y_{k} \in\right] x_{n_{k}-1} ; x_{n_{k}}\right]$ with $x_{p}=p \Delta$, one has

$$
f_{\{\lambda\}}\left(n_{1}, \ldots, n_{N}\right)=c^{-\frac{N}{2}} \mathrm{e}^{\frac{i \pi}{2} N} \varphi\left(y_{1}, \ldots, y_{N} \mid\left\{\lambda_{\ell_{a}}^{\mathrm{c}}\right\}_{1}^{N}\right) \cdot(1+\mathrm{O}(\Delta))
$$

and likewise

$$
f_{\{\mu\}}\left(n_{1}, \ldots, n_{N}\right)=c^{-\frac{N}{2}} \mathrm{e}^{\frac{i \pi}{2} N} \varphi\left(y_{1}, \ldots, y_{N} \mid\left\{\mu_{a}\right\}_{1}^{N}\right) \cdot(1+\mathrm{O}(\Delta)) .
$$

As a consequence,

$$
L_{1}=\int_{0}^{L} \overline{\varphi\left(y_{1}, \ldots, y_{N} \mid\left\{\mu_{a}\right\}\right)} \cdot \varphi\left(y_{1}, \ldots, y_{N} \mid\left\{\lambda_{\ell_{a}}^{\mathrm{c}}\right\}\right) \cdot g_{\Delta}\left(y_{1}, \ldots, y_{N}\right) \mathrm{d}^{N} y,
$$

with

$$
g_{\Delta}\left(y_{1}, \ldots, y_{N}\right)=\sum_{\substack{1 \leq n_{1}<\\ \cdots<n_{N} \leq M}} \prod_{k=1}^{N} \mathbf{1}_{\left.x_{n_{k}-1} ; x_{n_{k}}\right]}\left(y_{k}\right) \cdot(1+\mathrm{O}(\Delta)),
$$

where $\mathbf{1}_{] a ; b]}(x)$ denotes the indicator function of the interval ] $\left.a ; b\right]$.

It is readily seen that, for $\Delta$ small enough, $\sup _{[0 ; L]^{N}}\left|g_{\Delta}\right| \leq 2$, that $g_{\Delta} \in L^{1}\left([0 ; L]^{N}\right)$ and that, almost everywhere

$g_{\Delta}\left(y_{1}, \ldots, y_{N}\right) \rightarrow \mathbf{1}_{\mathcal{D}}\left(y_{1}, \ldots, y_{N}\right), \quad$ where $\mathcal{D}=\left\{\left(y_{1}, \ldots, y_{N}\right): 0 \leq y_{1}<\cdots<y_{N} \leq L\right\}$.

As both functions $\varphi$ are bounded on $[0 ; L]^{N}$, we are in position to apply the dominated convergence theorem: (A27) converges to the rhs of (3.1).

We have thus proven that the scalar product defined in terms of products of B operators and their adjoints do converge, in the $\Delta \rightarrow 0$ limit, to the scalar product of the continuous model. However, as follows from Theorem 2.1, such scalar products admits a finite-size $N$ determinant representation. It is straightforward to compute the $\Delta \rightarrow 0$ limit of the rhs in (2.28) hence obtaining the determinant representation for the scalar products in the continuous model.

\section{The form factors of the conjugated field operator}

In order to prove Theorem 3.2, we first notice that the restrictions of the operators $\chi_{k}$ and $\chi_{k}^{*}$ to the $N$-particle Hilbert space $\mathscr{H}_{N}=\operatorname{Vect}\left\{\chi_{n_{1}}^{*} \ldots \chi_{n_{N}}^{*}|0\rangle, 1 \leq n_{1} \leq \cdots \leq n_{N} \leq M\right\}$ are bounded operators

$\chi_{k}: \mathscr{H}_{N} \rightarrow \mathscr{H}_{N-1} \quad\left\|\chi_{k}\right\|_{N, N-1}=\sqrt{N \Delta}$ and $\chi_{k}: \mathscr{H}_{N} \rightarrow \mathscr{H}_{N+1} \quad\left\|\chi_{k}^{*}\right\|_{N, N+1}=\sqrt{(N+1) \Delta}$. 
Above, $\|\cdot\|_{N, N+1}$ stands for the operator norm on linear operators from $\mathscr{H}_{N}$ to $\mathscr{H}_{N+1}$. It then follows that, for $\Delta$ small enough,

$$
\left\|\tau^{-1}(v) \cdot B(v)-\beta_{M}^{*} / 2\right\|_{N, N+1}=\mathrm{O}\left(\Delta^{3 / 2}\right) .
$$

There, $\tau^{-1}(\nu) \cdot B(\nu)$ is given by (2.31) and $\beta_{k}^{*}$ is as defined in Lemma 1.1.

The bound (A31) follows from the fact that all the operators $\rho_{Z_{k}}$ are bounded on $\mathscr{H}_{N}$ and that they can be represented there, for $\Delta$ small enough, are uniformly convergent series. The rest follows from standard estimates of bounded operator-values series.

One can then represent the form factor as

$$
\mathcal{F}_{\{\lambda\} ;\{\mu\}}^{(\Delta)}=\left\langle\psi\left(\left\{\mu_{\ell_{a}}\right\}_{1}^{N+1}\right)\left|\tau^{-1}(\nu) B(v)\right| \psi\left(\left\{\lambda_{r_{a}}\right\}_{1}^{N}\right)\right\rangle=\mathcal{F}_{\{\lambda\} ;\{\mu\}}^{(\Delta, 1)}+\mathcal{F}_{\{\lambda\} ;\{\mu\}}^{(\Delta, 2)},
$$

where

$$
\mathcal{F}_{\{\lambda\} ;\{\mu\}}^{(\Delta, 2)}=\left\langle\psi\left(\left\{\mu_{\ell_{a}}\right\}_{1}^{N+1}\right)\left|\left\{\tau^{-1}(\nu) B(\nu)-\beta_{M}^{*} / 2\right\}\right| \psi\left(\left\{\lambda_{r_{a}}\right\}_{1}^{N}\right)\right\rangle
$$

and

$$
\mathcal{F}_{\{\lambda\} ;:\{\}}^{(\Delta, 1)}=\frac{1}{2}\left\langle\psi\left(\left\{\mu_{\ell_{a}}\right\}_{1}^{N+1}\right)\left|\beta_{M}^{*}\right| \psi\left(\left\{\lambda_{r_{a}}\right\}_{1}^{N}\right)\right\rangle .
$$

The Cauchy-Schwarz formula leads to

$$
\left|\mathcal{F}_{\{\lambda\} ;\{\mu\}}^{(\Delta, 2)}\right| \leq\left\|\psi\left(\left\{\mu_{\ell_{a}}\right\}_{1}^{N+1}\right)\right\| \cdot\left\|\psi\left(\left\{\lambda_{r_{a}}\right\}_{1}^{N}\right)\right\| \cdot\left\|\tau^{-1}(\nu) B(\nu)-\beta_{M}^{*} / 2\right\|_{N, N+1}=\mathrm{O}\left(\Delta^{\frac{3}{2}}\right) .
$$

There, we have used the results following from Sec. 2 of Appendix that norms of Bethe vectors are bounded uniformly in $\Delta$ small enough and the estimates (A31).

It remains to analyse the limit of $\left|\mathcal{F}_{\{\lambda\} ;\{\mu\}}^{(\Delta, 1)}\right|$. By computing the scalar products likewise to what has been done in Sec. 2 of Appendix, we obtain

$$
\mathcal{F}_{\{\lambda\} ;\{\}\}}^{(\Delta, 1)}=\frac{(\Delta c)^{N+1}}{2} \sum_{\substack{1 \leq n_{1} \leq \\ \cdots \leq n_{N} \leq M}} \overline{f_{\{\mu\}}}\left(n_{1}, \ldots, n_{N}, M\right) f_{\{\lambda\}}\left(n_{1}, \ldots, n_{N}\right) \prod_{a=1}^{M} \prod_{p=1}^{\# \Gamma_{a}-1} \frac{Z_{n_{a}}+p \Delta c / 4}{p+1} .
$$

Above, the sets $\Gamma_{a}$ are subordinate to the sequence of inegers $\left\{n_{1}, \ldots, n_{N}, M=n_{N+1}\right\}$. Very similar estimates and calculations to those gathered in Sec. 2 of Appendix, lead to the conclusion that

$$
\Delta^{-1} \cdot \mathcal{F}_{\{\lambda\} ;\{\mu\}}^{(\Delta, 1)} \underset{\Delta \rightarrow 0}{\longrightarrow}-\frac{i \sqrt{c}}{2} \int_{0}^{L} \overline{\varphi\left(y_{1}, \ldots, y_{N}, L \mid\left\{\mu_{\ell_{a}}^{\mathrm{c}}\right\}\right)} \cdot \varphi\left(y_{1}, \ldots, y_{N} \mid\left\{\lambda_{r_{a}}^{\mathrm{c}}\right\}\right) \mathbf{1}_{\mathcal{D}}\left(y_{1}, \ldots, y_{N}\right) \mathrm{d}^{N} y .
$$

Since, $\Delta^{-1} \mathcal{F}_{\{\lambda\} ;\{\{\}\}}^{(\Delta, 2)} \rightarrow 0$ in the $\Delta \rightarrow 0$ limit, we get that indeed, $\Delta^{-1} \mathcal{F}_{\{\lambda\} ;\{\mu\}}^{(\Delta)}$ does indeed converge to the form factor of the operator $-i \sqrt{c} \Phi^{\dagger}(0) / 2$ in the continuous model. The determinant representation for the form factor of the $\Phi^{\dagger}$ operator in the continuous model then follows from taking the $\Delta \rightarrow 0$ limit on the determinant representation given in Proposition 3.1, which is straightforward.

${ }^{1}$ Bytsko, A. G. and Teschner, J., "Quantization of models with non-compact quantum group symmetry: Modular XXZ magnet and lattice sinh-Gordon model," J. Phys. A 39, 12927-12982 (2006).

${ }^{2}$ Caux, J. -S., Glazman, L. I., Imambekov, A., and Shashi, A., "Exact asymptotes of static and dynamic correlation functions of the 1D Bose gas, (2010); e-print arXiv:cond-mat:1010.2268.

${ }^{3}$ Derkachov, S. E., Korchemsky, G. P., and Manashov, A. N., "Noncompact Heisenberg spin magnets from high-energy QCD: I. Baxter Q-operator and separation of variables," Nucl. Phys. B617137, 375-440 (2001).

${ }^{4}$ Destri, C. and DeVega, H. J., "Unified approach to Thermodynamic Bethe Ansatz and finite-size corrections for lattice models and field theories," Nucl. Phys. B 438, 413-454 (1995).

${ }^{5}$ Dorlas, T. C., "Orthogonality and completeness of the Bethe Ansatz eigenstates of the nonlinear Schrödinger model," Commun. Math. Phys. 154(2), 347-376 (1993).

${ }^{6}$ Izergin, A. G. and Korepin, V. E., "Lattice versions of quantum field theory models in two dimensions," Nucl. Phys. B 205, 401-413 (1982).

${ }^{7}$ Kitanine, N., Kozlowski, K. K., Maillet, J. -M., Slavnov, N. A., and Terras, V., "Thermodynamic limit of particle-hole form factors in the massless XXZ Heisenberg chain," e-print math-ph:1003.4557. 
${ }^{8}$ Kitanine, N., Kozlowski, K. K., Maillet, J. -M., Slavnov, N. A., and Terras, V., "Algebraic Bethe Ansatz approach to the asymptotics behavior of correlation functions," J. Stat. Mech.: Theory Exp. 04, P04003 (2009).

${ }^{9}$ Kitanine, N., Kozlowski, K. K., Maillet, J. -M., Slavnov, N. A., and Terras, V., "On the thermodynamic limit of form factors in the massless XXZ Heisenberg chain," J. Math. Phys. 50, 095209 (2009).

${ }^{10}$ Kojima, T., Korepin, V. E., and Slavonv, N. A., "Completely integrable equation for the quantum correlation functions of non-linear Schrödinger equation," Commun. Math. Phys. 189, 709-728 (1997).

${ }^{11}$ Korepin, V. E., "Calculation of norms of Bethe wave functions," Commun. Math. Phys. 86, 391-418 (1982).

${ }^{12}$ Korepin, V. E. and Slavnov, N. A., "The form factors in the finite volume," Int. J. Mod. Phys. B 13, 2933-2942 (1999).

${ }^{13}$ Kozlowski, K. K., "Large-distance and long-time asymptotic behavior of the reduced density matrix in the non-linear Schrödinger model," (2011); e-print:math-ph:11011626.

${ }^{14}$ Lieb, E. H. and Liniger, W., "Exact analysis of an interacting Bose gas. I: The general solution and the ground state," Phys. Rev. 130, 1605-1616 (1963).

${ }^{15}$ Niccoli, G., private communication (2011).

${ }^{16}$ Niccoli, G. and Teschner, J., "The Sine-Gordon model revisited I,” J. Stat. Mech. 1009, P09014 (2010).

${ }^{17}$ Oota, T., "Quantum projectors and local operators in lattice integrable models," J. Phys. A: Math. Gen. 37, 441-452 (2004).

${ }^{18}$ Ridout, D. and Teschner, J., "Integrability of a family of quantum field theories related to sigma models," (2011); e-print:hep-th: 11025716.

${ }^{19}$ Scheidmann, V., Introduction to Complex Analysis in Several Complex Variables (Birkhäuser Verlag, Basel, 2005).

${ }^{20}$ Simons, B., "Notes on infinite dimensional determinants," Adv. Math. 24, 244-273 (1977).

${ }^{21}$ Slavnov, N. A., "Calculation of scalar products of wave functions and form factors in the framework of the algebraic Bethe Ansatz," Theor. Math. Phys. 79, 502-508 (1989).

${ }^{22}$ Slavnov, N. A., "Non-equal time current correlation function in a one-dimensional Bose gas," Theor. Math. Phys. 82, 273-282 (1990).

${ }^{23}$ Teschner, J., "On the spectrum of the Sinh-Gordon model in finite volume," Nucl. Phys. B779, 403-429 (2008).

${ }^{24}$ Zamolodchikov, A. B. and Zamolodchikov, A. B., "Factorized S-matrices in two dimensions as the exact solutions of certain relativistic quantum field theory models," Ann. Phys. 120, 253-291 (1979). 